
= 


\title{
ACTIVE CONTROL OF ACOUSTIC PRESSURE FIELDS USING SMART MATERIAL TECHNOLOGIES ${ }^{1,2}$
}

\author{
H.T. Banks \\ Center for Research in Scientific Computation \\ North Carolina State University \\ Raleigh, NC 27695 \\ R.C. Smith \\ ICASE \\ NASA Langley Research Center \\ Hampton, VA 23681
}

\begin{abstract}
An overview describing the use of piezoceramic patches in reducing noise in a structural acoustics setting is presented. The passive and active contributions due to patches which are bonded to an Euler-Bernoulli beam or thin shell are briefly discussed and the results are incorporated into a 2-D structural acoustics model. In this model, an exterior noise source causes structural vibrations which in turn lead to interior noise as a result of nonlinear fluid/structure coupling mechanisms. Interior sound pressure levels are reduced via patches bonded to the flexible boundary (a beam in this case) which generate pure bending moments when an outof-phase voltage is applied. Well-posedness results for the infinite dimensional system are discussed and a Galerkin scheme for approximating the system dynamics is outlined. Control is implemented by using LQR optimal control theory to calculate gains for the linearized system and then feeding these gains back into the nonlinear system of interest. The effectiveness of this strategy for this problem is illustrated in an example.
\end{abstract}

\footnotetext{
${ }^{1}$ Invited Lecture, Workshop on Flow Control, Institute for Mathematics and Its Applications (IMA), University of Minnesota, November 16-20, 1992, Minneapolis, MN.

${ }^{2}$ The research of H.T.B. was supported in part by the Air Force Office of Scientific Research under grant AFOSR-90-0091. This research was also supported by the National Aeronautics and Space Administration under NASA Contract Numbers NAS1-18605 and NAS1-19480 while H.T.B. was a visiting scientist and R.C.S. was in residence at the Institute for Computer Applications in Science and Engineering (ICASE), NASA Langley Research Center, Hampton, VA 23681.
} 


\section{Introduction}

The recent development of highly fuel-efficient turboprop and turbofan engines which also produce high levels of interior cabin noise (especially at low frequencies) has stimulated a substantial effort on the development of a comprehensive active control methodology for interior pressure field cavities that have been excited by some primary or external source. In this overview paper, we shall discuss recent approaches and preliminary results in the growing effort to develop "smart" or "adaptive" material concepts (materials that possess the capability for both sensing and actuation are often called "smart" materials) and control strategies for such a comprehensive methodology.

Interior cavity noise in aircraft with turboprop engines is produced primarily through (nonlinear) fluid/structure interaction mechanisms. The turboprop blades produce an external acoustic pressure field which is converted into mechanical vibrations through fluid/structure interactions at the exterior aircraft cabin walls. In turn, these mechanical vibrations produce, through interactions of the interior cabin walls with the air in the cabin cavity, pressure waves or an interior acoustic pressure field.

Our discussion here focuses on a time domain state space approach to active or feedback control of noise in the interior acoustic cavity. We are especially interested in models and methodologies which treat transient dynamics. There is a substantial literature on active control of noise in a frequency domain setting (see $[18,21,24,26,28]$ for some examples and further references to both experimental and analytic efforts) as well as a growing literature on infinite dimensional state space time domain approaches (e.g. $[2,3,8,9,10])$. Earlier efforts by most researchers focused on a control methodology implemented through secondary source techniques with the input or secondary noise based on feedback of noise levels in the acoustic cavity. In this approach, a system of microphones and speakers is strategically placed in the interior cavity where one can sense the pressure field (composed of the primary source plus any secondary sources present). This information is used as feedback for the actuators or speakers which produce a (hopefully) optimally interfering signal (secondary noise) to reduce the total noise levels in certain critical zones (related to passenger comfort). Both frequency and time domain settings have been used in providing not only "proof of concept" analyses but also in designing and and implementing these ideas (to date, mainly in luxury class automobiles).

More recently, a second approach utilizing smart materials technology has captured the attention of investigators. There are a large number of classes of smart materials (e.g. electrorheological fluids, magnetostrictives, shape memory alloys) but we shall restrict our discussions in this paper to piezoceramic devices such as piezoceramic patches which, when bonded to a structure such as a beam, plate, or curved cylindrical shell, act as an electro-mechanical transducer. When excited by an electric field, the patch induces a strain in the material to which it is bonded and hence can be employed as an actuator. Moreover, if the host material undergoes a deformation (either bending or extension/contraction), this produces a strain in the patch which results in a voltage across the patch that is proportional to the strain and thereby permits the use of the patch as a mechanical sensor. If constructed and wired with proper circuits, these patches can be employed as "self-sensing actuators" [20], thereby providing a smart or adaptive material capability for the structure to which the device is bonded or in which it is embedded. When combined with a computational adaptive or feedback control element, the potential for self-controlled or intelligent structures is enormous. 
In our presentation and discussions of active control of noise, we shall concentrate on actuator aspects of piezoceramics. In the noise suppression example detailed below, we tacitly assume that acoustic pressure in the cavity and wall displacements and velocities are sensed for feedback. For a complete smart material system, one would use piezoceramic (strain) sensors and cavity pressure sensors to construct a state estimator for feedback.

The motivating example we consider consists of an exterior noise source which is separated from an interior cavity by an active wall or plate. This plate transmits noise or vibrations from the exterior field to the interior cavity via fluid/structure interactions thus leading to the formulation of a system of partial differential equations consisting of an acoustic wave equation coupled with elasticity equations for the plate. The control is implemented in the example via piezoceramic patches on the plate which are excited in a manner so as to produce pure bending moments. It should be noted that the incorporation of the feedback control in this manner leads to a system with an unbounded input term (in this case, a system with input coefficients involving the Dirac delta "function" and its "derivative"). Experiments are being designed and carried out at NASA Langley Research Center in which the interior cavity is taken to be cylindrical with a circular active plate to which sectorial piezoceramic patches are bonded.

While the motivating structural acoustics applications are three dimensional in nature, many of the theoretical and numerical issues concerning system modeling, the simulation of system dynamics, estimation of physical parameters, and the developments of feasible control strategies can be studied in 2-D geometries. In this work, we consider a 2-D domain $\Omega(t)$ which is bounded on three sides by hard walls and on the fourth by a flexible beam (see Figure 1). A periodic forcing function $f$, modeling an exterior noise source, causes vibrations in the beam which then lead to unwanted interior noise.

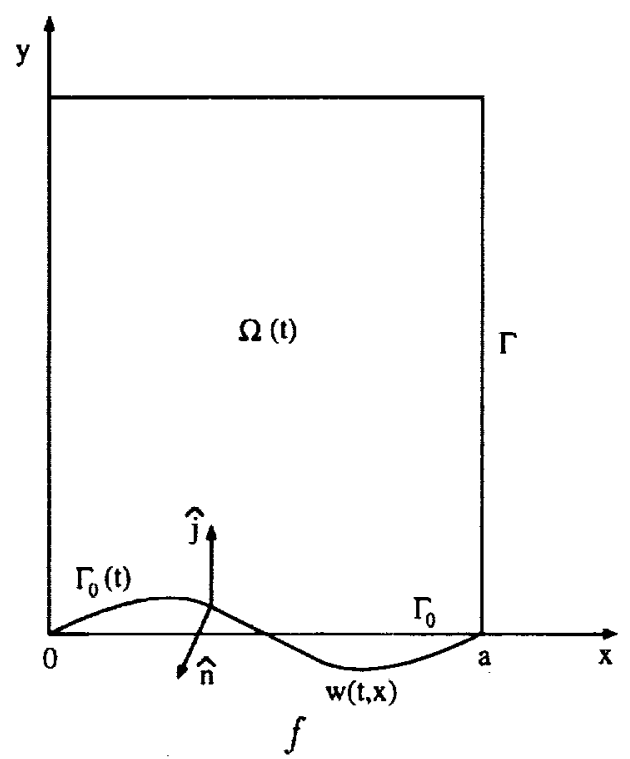

FIG. 1. The 2-D domain.

This specific problem was chosen since it is a two dimensional slice from a three dimensional cylindrical domain which models an experimental apparatus consisting of a rigid cylindrical pipe with a clamped aluminum plate at one end. 
As a 2-D analogue of the plate, the perturbable boundary $\Gamma_{0}(t)$ (see Figure 1) is modeled by a fixed-end Euler-Bernoulli beam having Kelvin-Voigt damping. Bonded to the beam are $s$ pairs of piezoceramic patches which are configured and excited in a manner so as to produce pure bending moments (see Figure 2). We reiterate that it is through the excitation of these patches that the sound pressure levels are controlled.

The acoustic response inside the cavity is modeled by a linear wave equation with zero normal velocity boundary conditions taken on three walls in order to simulate the rigid walls of the experimental pipe. The boundary conditions on the fourth (beam) side of the acoustic cavity result from nonlinear velocity and pressure couplings between the acoustic and structural responses (as discussed in [14], these coupling terms are nonlinear since they take place along the surface of the vibrating beam). Finally, under the assumption of small beam displacements which is inherent in the Euler-Bernoulli theory, the variable domain $\Omega(t)$ is replaced by the fixed domain $\Omega \equiv[0, a] \times[0, \ell]$ as shown in Figure 2 .

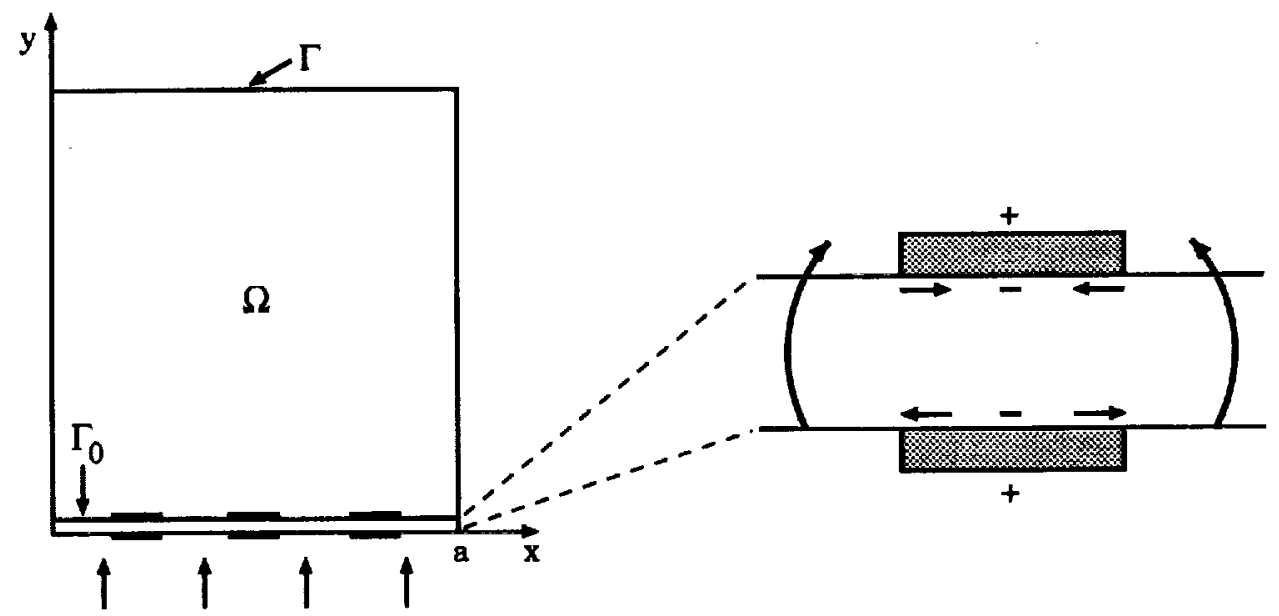

FIG. 2. Acoustic cavity with piezoceramic patches creating pure bending moments.

In terms of the velocity potential $\phi$ (so that $p=\rho_{f} \phi_{t}$ is the acoustic pressure) and the transverse beam displacements $w$, the strong form of the approximate controlled model for the coupled system is then given by

$$
\begin{aligned}
& \phi_{t t}=c^{2} \Delta \phi \quad, \quad(x, y) \in \Omega, t>0 \\
& \nabla \phi \cdot \hat{n}=0 \quad, \quad(x, y) \in \Gamma, t>0, \\
& \nabla \phi(t, x, w(t, x)) \cdot \hat{n}=w_{t}(t, x) \quad, \quad 0<x<a, t>0 \\
& \rho w_{t t}+\frac{\partial^{2} \mathcal{M}}{\partial x^{2}}=-\rho_{f} \phi_{t}(t, x, w(t, x))+f(t, x) \quad, \quad 0<x<a, t>0, \\
& w(t, 0)=\frac{\partial w}{\partial x}(t, 0)=w(t, a)=\frac{\partial w}{\partial x}(t, a)=0, \quad t>0, \\
& \phi(0, x, y)=\phi_{0}(x, y) \quad, \quad w(0, x)=w_{0}(x) \\
& \phi_{t}(0, x, y)=\phi_{1}(x, y) \quad, \quad w_{t}(0, x)=w_{1}(x)
\end{aligned}
$$


(for further details concerning the development of this model, see [14]). Here $\rho, \rho_{f}$ and $c$ are the beam density, equilibrium density of the atmosphere, and speed of sound in the cavity, respectively. The general beam moment $\mathcal{M}(t, x)$ consists of an internal component, depending on material and geometric properties of the beam and patches, and an external component (the control term) which results from the activation of the patches through an applied voltage. Specific descriptions of these moments in a variety of settings are given in the next section. Finally, the nonlinear coupling between the beam vibrations and the interior acoustic field manifests itself in the velocity term $\nabla \phi(t, x, w(t, x)) \cdot \hat{n}=w_{t}(t, x)$ and the backpressure $\rho_{f} \phi_{t}(t, x, w(t, x))$.

\section{Piezoceramic Patch/Structure Interactions}

As discussed in the last section, control is implemented in the system through the excitation of piezoceramic patches which are bonded to the beam. This affects the dynamics of the beam in two ways. The first effect is passive and results from the structural changes incurred with the bonding of the patches to the structure. In addition to the patch thickness, there is a nontrivial bonding layer, and both contribute to a moment of inertia which differs from that found in regions of the structure not covered with patches. Moreover, the density, Young's modulus and damping coefficient of the glue and patch differ from those of the beam, and as a result, these parameters must be modeled as piecewise constants in order to accurately match system frequencies (see [17]). The third passive contribution is due to the piezoelectric property which dictates that when the patch is subjected to an in-plane strain, a voltage proportional to the strain is produced. Hence longitudinal and transverse vibrations in the beam lead to the generation of current which provides additional damping in the structure. The final (active) contribution from the piezoceramic patches results from the in-plane strains which are produced when a voltage is applied. This leads to the generation of external moments and forces which enter the equations of motion as external loads.

The initial part of this section contains a discussion concerning the contributions due to patches which are bonded to an Euler-Bernoulli beam. The changes which are necessary for extending these arguments to plates and shells are then outlined in the latter part of the section with further details given in [16].

\subsection{Piezoceramic Patch/Beam Interactions}

In the discussion which follows, we consider an Euler-Bernoulli beam of length $\ell$, width $b$ and thickness $h$ as depicted in Figure 3. The Young's modulus, mass density (in mass per unit volume) and damping coefficient for the homogeneous beam are denoted by $E_{b}, \rho_{b}$ and $c_{D b}$, respectively. Bonded to the beam are piezoceramic patches which can be mounted either individually or in pairs as shown in Figures 3 and 4 . In the initial discussion concerning the contribution due to the patch pairs, it is assumed that both patches have thickness $T$, Young's modulus $E_{p e}$, density $\rho_{p e}$, and damping coefficient $c_{D p e}$. Moreover, it is assumed that the bonding layers for each patch have the same thickness, Young's modulus, density and damping coefficient, and these parameters are denoted by $T_{b \ell}, E_{b \ell}, \rho_{b \ell}$ and $c_{D b \ell}$, respectively. We emphasize that these assumptions are made solely for clarity of presentation, and similar 
results can be obtained in an analogous manner for the more general case in which the patches and bonding layers have differing thicknesses and material properties (see, for example, [16]).

For an Euler-Bernoulli beam having this configuration, force and moment balancing yields the strong form of the dynamic equations

$$
\begin{aligned}
& \rho(x) \frac{\partial^{2} u}{\partial t^{2}}-\frac{\partial N_{x}}{\partial x}=\hat{q}_{x} \\
& \rho(x) \frac{\partial^{2} w}{\partial t^{2}}+\frac{\partial^{2} M_{x}}{\partial x^{2}}=\hat{q}_{n}-\frac{\partial \hat{m}_{y}}{\partial x}
\end{aligned}
$$

where $N_{x}$ and $M_{x}$ are the internal force and moment resultants, respectively (see [12, 16]). As depicted in Figure 3, $w$ and $u$ denote the transverse and longitudinal displacements, respectively. The external surface loads $\hat{q}_{n}, \hat{q}_{x}$ and $\hat{m}_{y}$ denote normal forces, in-plane forces and moments, respectively. For patch pairs with edges at $x_{1}$ and $x_{2}$, the density of the structure is

$$
\rho(x)=\rho_{b} h b+2 b\left(\rho_{b \ell} T_{b \ell}+\rho_{p e} T\right) \chi_{p e}(x)
$$

where the characteristic function is given by

$$
\chi_{p e}(x)= \begin{cases}1, & x_{1} \leq x \leq x_{2} \\ 0, & \text { otherwise }\end{cases}
$$
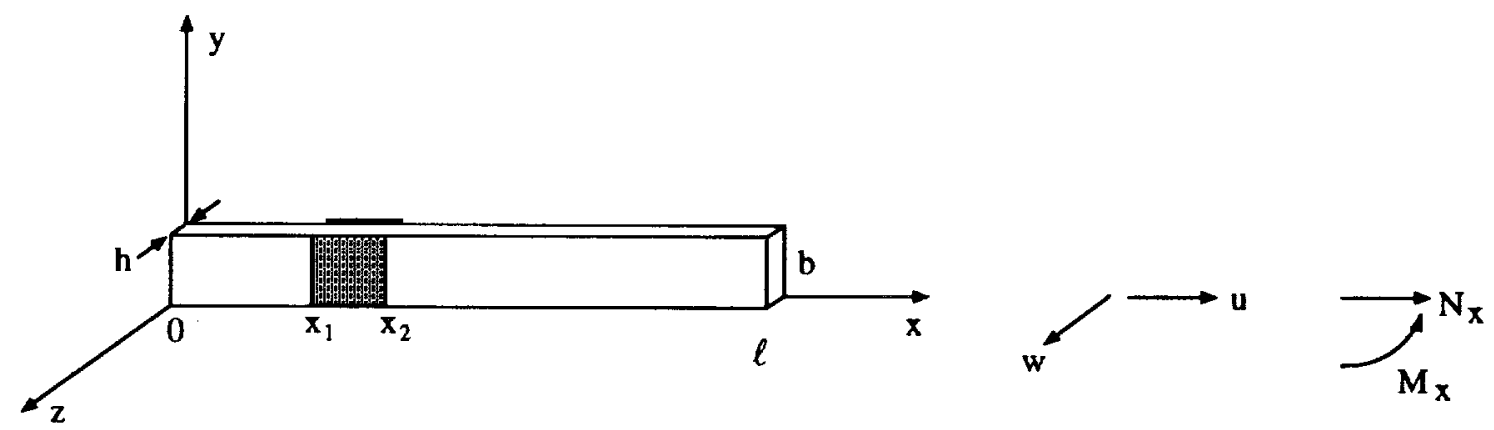

FIG. 3. Cantilever beam with piezoceramic patches.

A corresponding weak or variational form of the equations can be determined by choosing $V=H_{b}^{1}\left(\Gamma_{0}\right) \times H_{b}^{2}\left(\Gamma_{0}\right)$ for the space of trial functions where $\Gamma_{0}$ denotes the beam and the subscript $b$ again denotes the set of functions which must satisfy the essential boundary conditions. Through an energy derivation, one arrives at the variational form

$$
\begin{aligned}
& \int_{0}^{\ell}\left\{\rho(x) \frac{\partial^{2} u}{\partial t^{2}} \phi_{1}+N_{x} \frac{\partial \phi_{1}}{\partial x}-\hat{N}_{x} \frac{\partial \phi_{1}}{\partial x}\right\} d x=0 \quad \text { for all } \phi_{1} \in H_{b}^{1}\left(\Gamma_{0}\right) \\
& \int_{0}^{\ell}\left\{\rho(x) \frac{\partial^{2} w}{\partial t^{2}} \phi_{3}+M_{x} \frac{\partial^{2} \phi_{3}}{\partial x^{2}}-\hat{q}_{n} \phi_{3}-\hat{M}_{x} \frac{\partial^{2} \phi_{3}}{\partial x^{2}}\right\} d x=0 \quad \text { for all } \phi_{3} \in H_{b}^{2}\left(\Gamma_{0}\right)
\end{aligned}
$$

of the beam equations where $\hat{N}_{x}$ and $\hat{M}_{x}$ are external line force and moment resultants. As discussed in [16], the surface loads $\hat{q}_{x}$ and $\hat{m}_{y}$ of (2) are locally related to the forces and 
moments $\hat{N}_{x}$ and $\hat{M}_{x}$ (which are more natural quantities to use in a weak formulation) through the expressions $\hat{q}_{x}=-\frac{\partial \hat{N}_{x}}{\partial x}, \hat{m}_{y}=-\frac{\partial \hat{M}_{x}}{\partial x}$. Global expressions for the specific loads which result from the activation of the patches in both the strong and weak formulations are discussed later in the section.

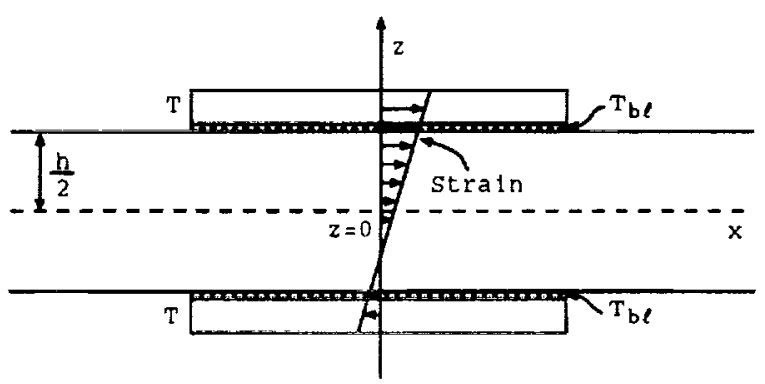

(a)

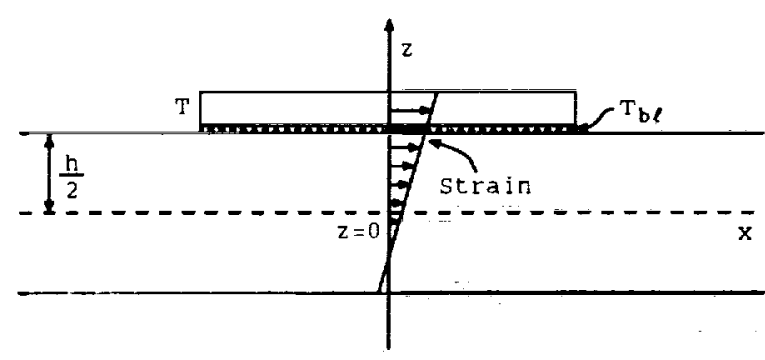

(b)

FIG. 4. Strain distribution for the composite structure undergoing bending and extension; (a) patch pair, and (b) single patch.

\section{Internal Moment and Force Resultants}

In order to determine expressions for the internal force and moment resultants $N_{x}$ and $M_{x}$, the patch pair configuration illustrated in Figure $4 \mathrm{a}$ is considered first. Because these resultants depend upon the stresses and ultimately upon the strains occurring in the structure, the description of the resultants begins with a description of the in-plane strains.

In accordance with the Euler-Bernoulli theory, the strain is assumed to be linear and is continuous throughout the combined structure. With $\varepsilon$ and $\kappa$ denoting the midsurface strain and change in curvature, respectively, the strain at an arbitrary point in the beam, bonding layer, or patch is given by $e=\varepsilon+\kappa z$ where $z$ is the distance of the point from the middle surface of the beam (see Figure 4a). Because of the differing Young's moduli and damping coefficients in the beam, bonding layer and patch, the stress slopes will differ in the various layers. Under the assumption that the stress is proportional to a linear combination of strain and strain rate, the stress is given by

$$
\sigma=\left\{\begin{array}{lll}
E_{b} e+c_{D b} \dot{e} & , & \text { beam } \\
E_{b \ell} e+c_{D b \ell} \dot{e} & , & \text { bonding layers } \\
E_{p e} e+c_{D p e} \dot{e} & , & \text { patches }
\end{array}\right.
$$

The coefficients $c_{D b}$ and $c_{D b \ell}$ are the Kelvin-Voigt damping coefficients for the beam and bonding layer while the coefficient $c_{D_{p e}}$ is taken to be a combination of the Kelvin-Voigt damping coefficient for the patch and the damping which results from the production of current when the structure vibrates. This latter contribution to the damping results from the piezoelectric effect of the patches which dictates that a voltage is produced when the patch is subjected to in-plane strains. Under the assumption that the Kelvin-Voigt (material) and electrical damping have approximately the same types of effect in the patch, we have

combined the two into the coefficient $c_{D p e}$ which is considered to be unknown and like the other 
parameters, must ultimately be estimated using data fitting techniques with experimental data when considering actual applications. We also point out that the expression (5) can easily be generalized to include the possibility of differing material properties in the two patches or bonding layers.

The force and moment resultants are obtained by integrating the stress across the thickness of the structure thus yielding the expressions

$$
\begin{array}{lll}
N_{x}=b \int_{-h / 2}^{h / 2} \sigma d z & M_{x}=b \int_{-h / 2}^{h / 2} \sigma z d z \quad & \text { regions without patches } \\
N_{x}=b \int_{-h / 2-T_{b \ell}-T}^{h / 2+T_{b \ell}+T} \sigma d z & , M_{x}=b \int_{-h / 2-T_{b \ell}-T}^{h / 2+T_{b \ell}+T} \sigma z d z \quad, \text { regions with patches. }
\end{array}
$$

The substitution of (5) into (6) yields expressions for the resultants in terms of the midsurface strain $\varepsilon$ and change in curvature $\kappa$. By considering infinitesimal deformations of the middle surface, $\varepsilon$ and $\kappa$ can be related to the longitudinal and transverse displacements $u$ and $w$ through the strain-displacement equations

$$
\varepsilon=\frac{\partial u}{\partial x} \quad, \quad \kappa=\frac{\partial^{2} w}{\partial x^{2}}
$$

(see [25], pages 9 and 46). For a beam having two patches bonded to it, the internal (material) force and moment resultants are then given by

$$
\begin{aligned}
& N_{x}=E h(x) \frac{\partial u}{\partial x}+c_{D} h(x) \frac{\partial^{2} u}{\partial x \partial t} \\
& M_{x}=E I(x) \frac{\partial^{2} w}{\partial x^{2}}+c_{D} I(x) \frac{\partial^{3} w}{\partial x^{2} \partial t}
\end{aligned}
$$

where

$$
\begin{array}{ll}
E h(x)=E_{b} h b+2 b\left[E_{b \ell} T_{b \ell}+E_{p e} T\right] \chi_{p e}(x) & , c_{D} h(x)=c_{D b} h b+2 b\left[c_{D b \ell} T_{b \ell}+c_{D_{p e}} T\right] \chi_{p e}(x) \\
E I(x)=E_{b} \frac{h^{3} b}{12}+\frac{2 b}{3}\left[E_{b \ell} a_{3 b \ell}+E_{p e} a_{3 p e}\right] \chi_{p e}(x), c_{D} I(x)=c_{D b} \frac{h^{3} b}{12}+\frac{2 b}{3}\left[c_{D b \ell} a_{3 b \ell}+c_{D p e} a_{3 p e}\right] \chi_{p e}(x) .
\end{array}
$$

Here $\chi_{p e}(x)$ again denotes the characteristic function described in (3), and the constants $a_{3 b \ell}$ and $a_{3 p e}$ are given by $a_{3 b \ell}=\left(h / 2+T_{b \ell}\right)^{3}-(h / 2)^{3}$ and $a_{3 p e}=\left(h / 2+T_{b \ell}+T\right)^{3}-\left(h / 2+T_{b \ell}\right)^{3}$.

The substitution of the force and moment resultants in (7) into the dynamic equations (2) yields the equations of motion for the combined structure in terms of the transverse and longitudinal displacements $w$ and $u$. As should be expected for a beam containing a pair of identical patches which are bonded symmetrically about the middle surface, the differential equations (under the first order Euler-Bernoulli assumptions) describing the vibrations in the two coordinate directions are uncoupled.

To see how this differs from the case in which a single patch is bonded to the beam, we now consider the case in which a patch of width $T$ is bonded to the beam over the region $x_{1} \leq x \leq x_{2}$ as shown in Figure $4 \mathrm{~b}$. 
Integrating the stresses through the combined thickness of the structure yields the resultant expressions

$$
\begin{aligned}
& N_{x}=E h(x) \frac{\partial u}{\partial x}+c_{D} h(x) \frac{\partial^{2} u}{\partial x \partial t}+E_{2}(x) \frac{\partial^{2} w}{\partial x^{2}}+c_{D 2}(x) \frac{\partial^{3} w}{\partial x^{2} \partial t} \\
& M_{x}=E I(x) \frac{\partial^{2} w}{\partial x^{2}}+c_{D} I(x) \frac{\partial^{3} w}{\partial x^{2} \partial t}+E_{2}(x) \frac{\partial u}{\partial x}+c_{D 2}(x) \frac{\partial^{2} u}{\partial x \partial t}
\end{aligned}
$$

The parameters in this case are given by

$$
\begin{array}{lll}
E h(x)=E_{b} h b+b\left[E_{b \ell} T_{b \ell}+E_{p e} T\right] \chi_{p e}(x) & , c_{D} h(x)=c_{D b} h b+b\left[c_{D b \ell} T_{b \ell}+c_{D p e} T\right] \chi_{p e}(x) \\
E I(x)=E_{b} \frac{h^{3} b}{12}+\frac{b}{3}\left[E_{b \ell} a_{3 b \ell}+E_{p e} a_{3 p e}\right] \chi_{p e}(x) & , c_{D} I(x)=c_{D b} \frac{h^{3} b}{12}+\frac{b}{3}\left[c_{D b \ell} a_{3 b \ell}+c_{D p e} a_{3 p e}\right] \chi_{p e}(x) \\
E_{2}(x)=\frac{b}{2}\left[E_{b \ell} a_{2 b \ell}+E_{p e} a_{2 p e}\right] \chi_{p e}(x) & , c_{D 2}(x)=\frac{b}{2}\left[c_{D b \ell} a_{2 b \ell}+c_{D p e} a_{2 p e}\right] \chi_{p e}(x)
\end{array}
$$

with $a_{3 b \ell}$ and $a_{3 p e}$ defined as before and $a_{2 b \ell}$ and $a_{2 p e}$ given by $a_{2 b \ell}=\left(h / 2+T_{b \ell}\right)^{2}-(h / 2)^{2}$, $a_{2 p e}=\left(h / 2+T_{b \ell}+T\right)^{2}-\left(h / 2+T_{b \ell}\right)^{2}$.

When the force and moment expressions in (8) are substituted into the dynamic equations (2), it is apparent that the longitudinal and transverse vibrations are coupled as a result of the asymmetry of the structure due to the single patch. This is in contrast to the case when patch pairs are bonded to the beam and helps to indicate the, in general, nontrivial effect that the patches have on the passive or material properties of the structure.

\section{External Moment and Force Resultants}

The second contribution from the piezoceramic patches is the generation of external moments and forces which results from the converse piezoelectric property that when a voltage is applied, in-plane strains are induced in the patch. The magnitude of these induced free strains is given by

$$
e_{p e_{1}}=\frac{d_{31}}{T} V_{1} \quad, \quad e_{p e_{2}}=\frac{d_{31}}{T} V_{2}
$$

where $d_{31}$ is a piezoceramic strain constant, and $V_{1}$ and $V_{2}$ are the voltages into the two patches in the pair. We point out that when a voltage is applied to a free patch with edge coordinates $x_{1}$ and $x_{2}$, the point $\bar{x}=\left(x_{1}+x_{2}\right) / 2$ will not move whereas the symmetric points on either side will move an equal amount in opposite directions. This motivates the use of the indicator function in several of the following definitions.

The stresses due to the excitation of the patches are given by

$$
\left(\sigma_{x}\right)_{p e_{1}}=-E_{p e} e_{p e_{1}} \quad, \quad\left(\sigma_{x}\right)_{p e_{2}}=-E_{p e} e_{p e_{2}}
$$

with the negative signs resulting from conservation of forces when balancing the material and induced stresses in the patch.

The integration of these stresses through the thickness of the patches yields the expressions

$$
\begin{aligned}
& \left(M_{x}\right)_{p e}=\left[\left(M_{x}\right)_{p e_{1}}+\left(M_{x}\right)_{p e_{2}}\right] \chi_{p e}(x) \\
& \left(N_{x}\right)_{p e}=\left[\left(N_{x}\right)_{p e_{1}}+\left(N_{x}\right)_{p e_{2}}\right] \chi_{p e}(x) S_{1,2}(x)
\end{aligned}
$$


where

$$
\begin{array}{lll}
\left(M_{x}\right)_{p e_{1}}=-\frac{1}{2} E_{p e} b d_{31}\left(h+2 T_{b \ell}+T\right) V_{1} & & \left(M_{x}\right)_{p e_{2}}=\frac{1}{2} E_{p e} b d_{31}\left(h+2 T_{b \ell}+T\right) V_{2} \\
\left(N_{x}\right)_{p e_{1}}=-E_{p e} b d_{31} V_{1} & , & \left(N_{x}\right)_{p e_{2}}=-E_{p e} b d_{31} V_{2}
\end{array}
$$

for the external moments and forces generated by the activation of the patches. The presence of the indicator function

$$
S_{1,2}(x)=\left\{\begin{array}{cll}
1 & , & x<\left(x_{1}+x_{2}\right) / 2 \\
0 & , & x=\left(x_{1}+x_{2}\right) / 2 \\
-1 & , & x>\left(x_{1}+x_{2}\right) / 2
\end{array}\right.
$$

results from the fact that for homogeneous patches having uniform thickness, opposite but equal strains are generated about the point $\bar{x}=\left(x_{1}+x_{2}\right) / 2$.

These expressions can then be substituted directly into the weak equations (4) as loads on the beam (with $\hat{q}_{n}=0$ and $\hat{N}_{x}=\left(N_{x}\right)_{p e}, \hat{M}_{x}=\left(M_{x}\right)_{p e}$ ). In order to determine the patch loads for the strong form of the beam equations, the corresponding surface moments and forces are found via the relationships

$$
\hat{q}_{x}=-S_{1,2}(x) \frac{\partial\left(N_{x}\right)_{p e}}{\partial x} \quad, \quad \hat{m}_{y}=-\frac{\partial\left(M_{x}\right)_{p e}}{\partial x}
$$

and these latter values are used in (2). We point out that this results in the need to differentiate across discontinuities in characteristic and indicator functions (once for the force and twice for the moment) whereas this problem is avoided in the weak formulation since the derivatives are transferred on the test functions. In fact, the effect of the characteristic functions in the latter case is to simply restrict the integrals to the region covered by the patches.

The general moments in the beam component of the structural acoustic system (1) can now be described in terms of the internal and external moments just discussed. By combining both the passive and active contributions due to a single pair of patches which are excited out-of-phase, the general moment is given by

$$
\mathcal{M}=M_{x}-\left(M_{x}\right)_{p e}
$$

where the internal and external moments are

$$
\begin{aligned}
& M_{x}=E I(x) \frac{\partial^{2} w}{\partial x^{2}}+c_{D} I(x) \frac{\partial^{3} w}{\partial x^{2} \partial t} \\
& \left(M_{x}\right)_{p e}=E_{p e} b d_{31}\left(h+2 T_{b \ell}+T\right) V \chi_{p e}(x)=\mathcal{K}^{B} V \chi_{p e}(x)
\end{aligned}
$$

as given in (7) and (9), respectively (the latter expression is obtained by taking $V=V_{1}=-V_{2}$ in (9)). We emphasize that the out-of-phase excitation of the patches produces pure bending moments and hence only transverse vibrations are present in the beam response.

For a system in which $s$ pairs of patches are bonded to a beam and are excited out-of-phase, the beam component of the system (1) has the form

$$
\rho(x) \frac{\partial^{2} w}{\partial t^{2}}+\frac{\partial^{2}}{\partial x^{2}}\left(E I(x) \frac{\partial^{2} w}{\partial x^{2}}+c_{D} I(x) \frac{\partial^{3} w}{\partial x^{2} \partial t}\right)+\rho_{f} \phi_{t}(w)=f+\sum_{i=1}^{s} \mathcal{K}_{i}^{B} u_{i}(t) \frac{d^{2}}{d x^{2}} \chi_{p e_{i}}(x)
$$


where $\chi_{p e_{i}}(x)$ denotes the characteristic function over the $i^{t h}$ patch pair and $u_{i}(t)$ is the voltage into the $i^{t h}$ pair. The parameters $E I$ and $c_{D} I$ are given by

$$
\begin{aligned}
& E I(x)=E_{b} \frac{h^{3} b}{12}+\sum_{i=1}^{s} \frac{2 b}{3}\left[E_{b \ell_{i}} a_{3 b \ell_{i}}+E_{p e_{\mathrm{i}}} a_{3 p e_{i}}\right] \chi_{p e_{\mathrm{i}}}(x) \\
& c_{D} I(x)=c_{D b} \frac{h^{3} b}{12}+\sum_{i=1}^{s} \frac{2 b}{3}\left[c_{D b \ell_{\mathrm{i}}} a_{3 b \ell_{i}}+c_{D p e_{\mathrm{i}}} a_{3 p e_{\mathrm{i}}}\right] \chi_{p e_{\mathrm{i}}}(x)
\end{aligned}
$$

while the patch parameters are given by $\mathcal{K}_{i}^{B}=E_{p e_{i}} b d_{31_{i}}\left(h+2 T_{b \ell_{i}}+T_{i}\right)$ (in these definitions, the bonding layers and patches in the $i^{\text {th }}$ pair are considered to have thickness $T_{b \ell_{i}}$ and $T_{i}$, respectively). We note that the discontinuous parameters $\rho, E I, c_{D} I$ and $\mathcal{K}_{i}^{B}$ lead to second derivatives of characteristic functions which causes difficulties in the strong form of the equations. The transfer of these derivatives onto test functions eliminates these problems in the weak form of the equations and is one motivation for using the weak form of the system equations as discussed in the next section.

\subsection{Patch Contributions to Plate and Shell Dynamics}

In the first part of this section, the contributions from piezoceramic patches to the longitudinal and transverse vibrations of an Euler-Bernoulli beam were examined. It was noted that the patch contributions could be categorized into two types; the first resulted from the structural changes incurred when the patches were bonded to the beam while the second effect was due to the activation of the patches when a voltage was applied. These same types of effects result when piezoceramic patches are bonded to more complex structures such as thin plates or shells.

The motion of a plate differs from that of a beam in that two sets of longitudinal motion are present with the stretching in one coordinate direction related to the contraction in the other through the Poisson ratio $\nu$. In thin shells, the transverse and longitudinal vibrations are coupled due to the underlying curvature of the structure. However, once the underlying dynamic equations in terms of the force and moment resultants are known, the effects due to the presence and activation of the piezoceramic patches can be determined in a manner analogous to that discussed above for thin beams (see [16]).

To illustrate, we consider a thin circular cylindrical shell of radius $R$, thickness $h$ and having the axial coordinate $x$ as shown in Figure 5. As in the beam discussion, the variable $z$

measures the distance of a point on the structure from the corresponding point on the middle surface $(z=0)$ along the normal to the middle surface. 


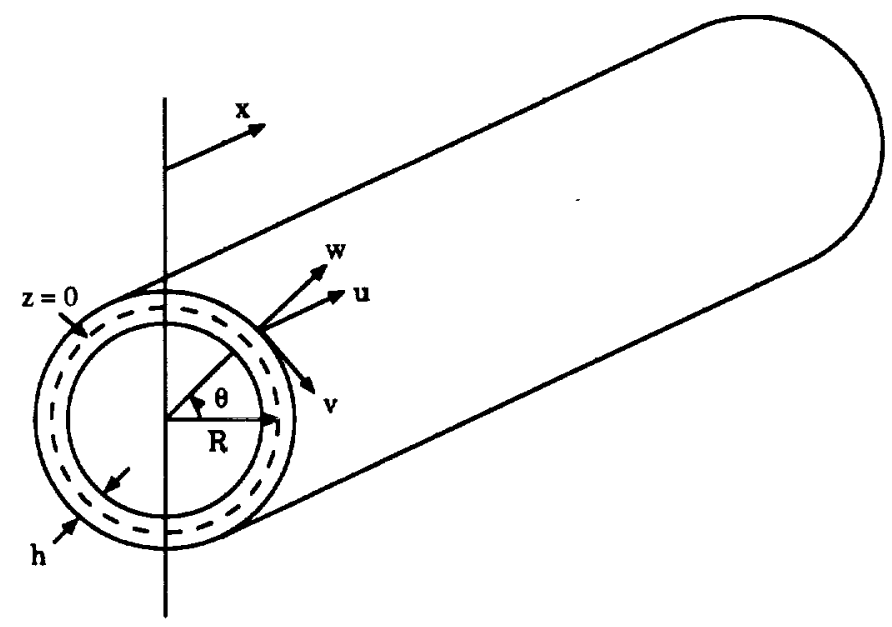

FIG. 5. The thin cylindrical shell.

As discussed in [16], the infinitesimal strain relationships for a cylindrical shell are

$$
e_{x}=\varepsilon_{x}+z \kappa_{x} \quad, \quad e_{\theta}=\frac{1}{1+z / R}\left(\varepsilon_{\theta}+z \kappa_{\theta}\right) \quad, \quad \gamma_{x \theta}=\frac{1}{1+z / R}\left[\varepsilon_{x \theta}+z\left(1+\frac{z}{2 R}\right) \tau\right]
$$

where $e_{x}$ and $e_{\theta}$ are normal strains at an arbitrary point within the cylindrical shell and $\gamma_{x \theta}$ is the shear strain. Here $\varepsilon_{x}, \varepsilon_{\theta}$ and $\varepsilon_{x \theta}$ are the normal and shear strains in the middle surface and $\kappa_{x}, \kappa_{\theta}$ and $\tau$ are the midsurface changes in curvature and midsurface twist (see [23], page 8).

In terms of the axial, tangential and radial displacements $u, v$ and $w$, respectively, the expressions for the midsurface strains and changes in curvature for the cylindrical shell are

$$
\begin{array}{llll}
\varepsilon_{x}=\frac{\partial u}{\partial x} & \varepsilon_{\theta}=\frac{1}{R} \frac{\partial v}{\partial \theta}+\frac{w}{R} & , \quad \varepsilon_{x \theta}=\frac{\partial v}{\partial x}+\frac{1}{R} \frac{\partial u}{\partial \theta} \\
\kappa_{x}=-\frac{\partial^{2} w}{\partial x^{2}} & , \quad \kappa_{\theta}=-\frac{1}{R^{2}} \frac{\partial^{2} w}{\partial \theta^{2}}+\frac{1}{R^{2}} \frac{\partial v}{\partial \theta} & , \tau=-\frac{2}{R} \frac{\partial^{2} w}{\partial x \partial \theta}+\frac{2}{R} \frac{\partial v}{\partial x} .
\end{array}
$$

If a generalized Hooke's law in which stress is assumed to be proportional to a linear combination of strain and strain rate is used as the constitutive relation, the stresses in the shell are given by

$$
\begin{aligned}
& \sigma_{x}=\frac{E_{s}}{1-\nu_{s}^{2}}\left(e_{x}+\nu_{s} e_{\theta}\right)+\frac{c_{D_{s}}}{1-\nu_{s}^{2}}\left(\dot{e}_{x}+\nu_{s} \dot{e}_{\theta}\right) \\
& \sigma_{\theta}=\frac{E_{s}}{1-\nu_{s}^{2}}\left(e_{\theta}+\nu_{s} e_{x}\right)+\frac{c_{D_{s}}}{1-\nu_{s}^{2}}\left(\dot{e}_{\theta}+\nu_{s} \dot{e}_{x}\right) \\
& \sigma_{x \theta}=\sigma_{\theta x}=\frac{E_{s}}{2\left(1+\nu_{s}\right)} \gamma_{x \theta}+\frac{c_{D_{s}}}{2\left(1+\nu_{s}\right)} \dot{\gamma}_{x \theta}
\end{aligned}
$$

where $\sigma_{x}$ and $\sigma_{\theta}$ are normal stresses and $\sigma_{x \theta}$ and $\sigma_{\theta x}$ are tangential shear stresses. The constants $E_{s}, \nu_{s}$ and $c_{D_{s}}$ are the Young's modulus, Poisson ratio, and damping coefficient for the shell. Similar relations are found in the bonding layers and patches (see (5) for analogous expressions for the beam). 
The internal or material moment and force resultants are obtained by integrating the stresses across the thickness of the structure. For patches having thickness $T$ and bonding layers of thickness $T_{b \ell}$, this yields the expressions

$$
\begin{aligned}
& {\left[\begin{array}{l}
N_{x} \\
N_{x \theta}
\end{array}\right]=\int_{-h / 2-T_{b \ell}-T}^{h / 2+T_{b \ell} T}\left[\begin{array}{l}
\sigma_{x} \\
\sigma_{x \theta}
\end{array}\right]\left(1+\frac{z}{R}\right) d z \quad, \quad\left[\begin{array}{c}
N_{\theta} \\
N_{\theta x}
\end{array}\right]=\int_{-h / 2-T_{b \ell}-T}^{h / 2+T_{b \ell}+T}\left[\begin{array}{l}
\sigma_{\theta} \\
\sigma_{\theta x}
\end{array}\right] d z} \\
& {\left[\begin{array}{l}
M_{x} \\
M_{x \theta}
\end{array}\right]=\int_{-h / 2-T_{b \ell}-T}^{h / 2+T_{b \ell}+T}\left[\begin{array}{l}
\sigma_{x} \\
\sigma_{x \theta}
\end{array}\right]\left(1+\frac{z}{R}\right) z d z \quad, \quad\left[\begin{array}{l}
M_{\theta} \\
M_{\theta x}
\end{array}\right]=\int_{-h / 2-T_{b \ell}-T}^{h / 2+T_{b \ell}+T}\left[\begin{array}{l}
\sigma_{\theta} \\
\sigma_{\theta x}
\end{array}\right] z d z}
\end{aligned}
$$

in regions of the structure covered by the patches with similar expressions in those region of the structure consisting solely of shell material (the limits of integration in this latter case are $-h / 2$ and $h / 2$ ). Explicit descriptions for these internal moment and force resultants can be found in [16].

In a shell which is excited by the activation of piezoceramic patches, the external moments and forces are due to the in-plane strains

$$
e_{p e_{1}}=\left(e_{x}\right)_{p e_{1}}=\left(e_{\theta}\right)_{p e_{1}}=\frac{d_{31}}{T} V_{1} \quad, \quad e_{p e_{2}}=\left(e_{x}\right)_{p e_{2}}=\left(e_{\theta}\right)_{p e_{2}}=\frac{d_{31}}{T} V_{2}
$$

which result from the input of the voltages $V_{1}$ and $V_{2}$ into the outer and inner patches. The resulting external stresses are given by

$$
\left(\sigma_{x}\right)_{p e_{1}}=\left(\sigma_{\theta}\right)_{p e_{1}}=-\frac{E_{1}}{1-\nu_{1}} e_{p e_{1}} \quad, \quad\left(\sigma_{x}\right)_{p e_{2}}=\left(\sigma_{\theta}\right)_{p e_{2}}=-\frac{E_{2}}{1-\nu_{2}} e_{p e_{2}} .
$$

For a patch with bounding values $x_{1}, x_{2}, \theta_{1}$ and $\theta_{2}$ the total external line moments and forces are

$$
\begin{aligned}
& \left(M_{x}\right)_{p e}=\left[\left(M_{x}\right)_{p e_{1}}+\left(M_{x}\right)_{p e_{2}}\right] \chi_{p e}(x, \theta) \\
& \left(M_{\theta}\right)_{p e}=\left[\left(M_{\theta}\right)_{p e_{1}}+\left(M_{\theta}\right)_{p e_{2}}\right] \chi_{p e}(x, \theta) \\
& \left(N_{x}\right)_{p e}=\left[\left(N_{x}\right)_{p e_{1}}+\left(N_{x}\right)_{p e_{2}}\right] \chi_{p e}(x, \theta) S_{1,2}(x) \hat{S}_{1,2}(\theta) \\
& \left(N_{\theta}\right)_{p e}=\left[\left(N_{\theta}\right)_{p e_{1}}+\left(N_{\theta}\right)_{p e_{2}}\right] \chi_{p e}(x, \theta) S_{1,2}(x) \hat{S}_{1,2}(\theta)
\end{aligned}
$$

where the indicator function $S_{1,2}(x)$ is defined in (10) (with a similar definition for $\hat{S}_{1,2}(\theta)$ ) and

$$
\chi_{p e}(x, \theta)= \begin{cases}1, & x_{1} \leq x \leq x_{2}, \theta_{1} \leq \theta \leq \theta_{2} \\ 0, & \text { otherwise }\end{cases}
$$

The individual patch moments are obtained by integrating the external stress distribution through the thickness of the patches in the same manner used in the beam analysis (see [16]).

In order to obtain a strong form of the equations of motion, force and moment balancing 
can be used to obtain Donnell-Mushtari shell equations

$$
\begin{gathered}
R \rho(x, \theta) \frac{\partial^{2} u}{\partial t^{2}}-R \frac{\partial N_{x}}{\partial x}-\frac{\partial N_{\theta x}}{\partial \theta}=-R \frac{\partial\left(N_{x}\right)_{p e}}{\partial x} S_{1,2}(x) \hat{S}_{1,2}(\theta) \\
R \rho(x, \theta) \frac{\partial^{2} v}{\partial t^{2}}-\frac{\partial N_{\theta}}{\partial \theta}-R \frac{N_{x \theta}}{\partial x}=-\frac{\partial\left(N_{\theta}\right)_{p e}}{\partial \theta} S_{1,2}(x) \hat{S}_{1,2}(\theta) \\
R \rho(x, \theta) \frac{\partial^{2} w}{\partial t^{2}}-R \frac{\partial^{2} M_{x}}{\partial x^{2}}-\frac{1}{R} \frac{\partial^{2} M_{\theta}}{\partial \theta^{2}}-2 \frac{\partial^{2} M_{x \theta}}{\partial x \partial \theta}+N_{\theta}=R \hat{q}_{n}-R \frac{\partial^{2}\left(M_{x}\right)_{p e}}{\partial x}-\frac{1}{R} \frac{\partial\left(M_{\theta}\right)_{p e}}{\partial \theta}
\end{gathered}
$$

(see $[16,23]$ for a more detailed derivation of these equations as well as a discussion concerning the assumptions that are made in obtaining this and other forms of the equations of motion for a thin shell). The contributions due to the patches are incorporated in the internal moments and forces (11), the external moments and forces (12), and the variable density $\rho(x, \theta)$.

\section{Weak Form and Well-Posedness of the Structural Acoustics Model}

As discussed in the last two sections, the incorporation of the piezoceramic patch contributions into the strong form of the modeling system equations leads to first and second derivatives of characteristic functions since both the internal and external moments contain discontinuities at the edges of the patches. This yields an unbounded control input operator and leads to difficulties when approximating the dynamics of the coupled system. To avoid these difficulties, it is advantageous to formulate the problem in weak or variational form (the use of the variational form also permits the use of basis functions having less smoothness than those used when approximating the solution to the strong form of the equations).

\subsection{Weak Form of the System Equations}

The state for the second-order form of the 2-D structural acoustics problem is taken to be $z=(\phi, w)$ in the Hilbert space $H=\bar{L}^{2}(\Omega) \times L^{2}\left(\Gamma_{0}\right)$ with the energy inner product

$$
\left\langle\left(\begin{array}{c}
\phi \\
w
\end{array}\right),\left(\begin{array}{c}
\xi \\
\eta
\end{array}\right)\right\rangle_{H}=\int_{\Omega} \frac{\rho_{f}}{c^{2}} \phi \xi d \omega+\int_{\Gamma_{0}} \rho_{b} w \eta d \gamma .
$$

The choice of the space $\bar{L}^{2}(\Omega)$, defined as the quotient of $L^{2}(\Omega)$ over the constant functions, results from the fact that the potentials are determined only up to a constant.

To provide a class of functions which are considered when defining a variational form of the problem, we also define the Hilbert space $V=\bar{H}^{1}(\Omega) \times H_{0}^{2}\left(\Gamma_{0}\right)$ where $\bar{H}^{1}(\Omega)$ is the quotient space of $H^{1}$ over the constant functions and $H_{0}^{2}\left(\Gamma_{0}\right)$ is given by $H_{0}^{2}\left(\Gamma_{0}\right)=$ $\left\{\psi \in H^{2}\left(\Gamma_{0}\right): \psi(x)=\psi^{\prime}(x)=0\right.$ at $\left.x=0, a\right\}$. The $V$ inner product is taken as (here and below we use the notation $D=\frac{\partial}{\partial x}$ )

$$
\left\langle\left(\begin{array}{c}
\phi \\
w
\end{array}\right),\left(\begin{array}{l}
\xi \\
\eta
\end{array}\right)\right\rangle_{V}=\int_{\Omega} \rho_{f} \nabla \phi \cdot \nabla \xi d \omega+\int_{\Gamma_{0}} E I D^{2} w D^{2} \eta d \gamma
$$


As discussed in [14], integration in combination with the use of Green's theorem then yields the nonlinear first-order variational form

$$
\begin{aligned}
\int_{\Omega} \frac{\rho_{f}}{c^{2}} \phi_{t t} \xi d \omega & +\int_{\Gamma_{0}} \rho w_{t t} \eta d \gamma \\
& +\int_{\Omega} \rho_{f} \nabla \phi \cdot \nabla \xi d \omega+\int_{\Gamma_{0}} E I D^{2} w D^{2} \eta d \gamma \\
& +\int_{\Gamma_{0}}\left\{c_{D} I D^{2} w_{t} D^{2} \eta+\rho_{f}\left[\phi_{t}(w) \eta-w_{t} \xi\right]\right\} d \gamma \\
& =\int_{\Gamma_{0}} \sum_{i=1}^{s} \mathcal{K}_{i}^{B} u_{i}(t) \chi_{p e_{i}}(x) D^{2} \eta d \gamma+\int_{\Gamma_{0}} f \eta d \gamma
\end{aligned}
$$

for all $(\xi, \eta)$ in $V$ (here $\chi_{p e_{i}}(x)$ denotes the characteristic function over the $i^{t h}$ patch). We note that the nonlinear coupling term can be written as $\phi_{t}(t, x, w(t, x))=\phi_{t}(t, x, 0)+\tilde{\phi}_{t}(t, x, w(t, x))$ where $\tilde{\phi}_{t}(t, x, w(t, x)) \equiv \phi_{t}(t, x, w(t, x))-\phi_{t}(t, x, 0)$. We will make use of this decomposition in the abstract formulation of the nonlinear system as a perturbation of a linearized system in our discussion below. Again, a more complete discussion and motivation concerning the formulation of the first-order system in weak form is given in [14].

We point out that in this variational form the derivatives have been transferred from the plate and patch moments onto the test functions. This eliminates the problem of having to approximate the derivatives of the characteristic function and the Dirac delta as is the case with the strong form of the equations.

The system (13) can be formally approximated by replacing the state variables by their finite dimensional approximations and constructing the resulting matrix system. Hence it is in a form which is suitable for use in applications. In order to discuss the well-posedness of the model, however, it is advantageous to pose the problem in terms of sesquilinear forms and the bounded operators which they define, and this is the subject of the rest of the section.

\subsection{Abstract First-Order Formulation}

As motivated by the theoretical results in $[3,4,6,15]$, we consider (see [29] for basic definitions and fundamental functional analysis theory) the Gelfand triple $V \hookrightarrow H \simeq H^{*} \hookrightarrow V^{*}$ with pivot space $H$ and define sesquilinear forms $\sigma_{i}: V \times V \rightarrow \mathbb{R}, i=1,2$ by

$$
\begin{gathered}
\sigma_{1}(\Phi, \Psi)=\int_{\Omega} \rho_{f} \nabla \phi \cdot \nabla \xi d \omega+\int_{\Gamma_{0}} E I D^{2} w D^{2} \eta d \gamma, \\
\sigma_{2}(\Phi, \Psi)=\int_{\Gamma_{0}}\left\{c_{D} I D^{2} w D^{2} \eta+\rho_{f}(\phi \eta-w \xi)\right\} d \gamma
\end{gathered}
$$

where $\Phi=(\phi, w)$ and $\Psi=(\xi, \eta)$ are in $V$.

As detailed for a similar problem in [15], it is straightforward to show that with these definitions, $\sigma_{1}$ and $\sigma_{2}$ are bounded (there exist $c_{1}$ and $c_{2}$ such that $\left|\sigma_{1}(\Phi, \Psi)\right| \leq c_{1}|\Phi|_{V}|\Psi|_{V}$ and $\left.\left|\sigma_{2}(\Phi, \Psi)\right| \leq c_{2}|\Phi|_{V}|\Psi|_{V}\right), \sigma_{1}$ is V-elliptic and $\sigma_{2}$ is H-semielliptic (there exist $c>0$ and $b \geq 0$ such that $\operatorname{Re} \sigma_{1}(\Phi, \Phi) \geq c|\Phi|_{V}^{2}$ and $\operatorname{Re} \sigma_{2}(\Phi, \Phi) \geq b|\Phi|_{H}^{2}$ for all $\left.\Phi \in V\right)$ and that $\sigma_{1}$ is 
symmetric $\left(\sigma_{1}(\Phi, \Psi)=\sigma_{1}(\Psi, \Phi)\right.$ for all $\left.\Phi, \Psi \in V\right)$. As a result of the boundedness, we can define operators $A_{1}, A_{2} \in \mathcal{L}\left(V, V^{*}\right)$ by

$$
\left\langle A_{i} \Phi, \Psi\right\rangle_{V^{*}, V}=\sigma_{i}(\Phi, \Psi)
$$

for $i=1,2$.

To account for the control contributions, we let $U$ denote the Hilbert space containing the control inputs $(U=\mathbb{R}$ in our structural acoustics example), and we define the control operator $B \in \mathcal{L}\left(U, V^{*}\right)$ by

$$
\langle B u, \Psi\rangle_{V^{*}, V}=\int_{\Gamma_{0}} \sum_{i=1}^{s} \mathcal{K}_{i}^{B} u_{i} \chi_{p e_{i}}(x) D^{2} \eta d \gamma
$$

for $\Psi \in V$, where $\langle\cdot,\rangle_{V^{*}, V}$ is the usual duality pairing. Finally, letting $F=\left(0, f / \rho_{b}\right)$ and $G\left(z, z_{t}\right)=\left(0,-\rho_{f} \tilde{\phi}_{t}(w)\right)$ where again, $\tilde{\phi}_{t}(w)=\tilde{\phi}_{t}(t, x, w(t, x))=\phi_{t}(t, x, w(t, x))-\phi_{t}(t, x, 0)$ denotes the nonlinear perturbation to the linear coupling term, we can write the control system in weak or variational form

$$
\left\langle z_{t t}(t), \Psi\right\rangle_{V^{*}, V}+\sigma_{2}\left(z_{t}(t), \Psi\right)+\sigma_{1}(z(t), \Psi)=\left\langle B u(t)+F(t)+G\left(z(t), z_{t}(t)\right), \Psi\right\rangle_{V^{*}, V}
$$

for $\Psi$ in $V$. This then yields the system

$$
z_{t t}(t)+A_{2} z_{t}(t)+A_{1} z(t)=B u(t)+F(t)+G\left(z(t), z_{t}(t)\right)
$$

in $V^{*}$.

To apply infinite dimensional control results for periodic forcing functions to this problem, it is advantageous to write the system in first-order form. This is accomplished by defining. the product spaces $\mathcal{H}=V \times H$ and $\mathcal{V}=V \times V$ with the norms

$$
\begin{aligned}
& |(\Phi, \Psi)|_{\mathcal{H}}^{2}=|\Phi|_{V}^{2}+|\Psi|_{H}^{2}, \\
& |(\Phi, \Psi)|_{V}^{2}=|\Phi|_{V}^{2}+|\Psi|_{V}^{2} .
\end{aligned}
$$

We point out that $\mathcal{V} \hookrightarrow \mathcal{H} \simeq \mathcal{H}^{*} \hookrightarrow \mathcal{V}^{*}$ again forms a Gelfand triple.

The sesquilinear form $\sigma: \mathcal{V} \times \mathcal{V} \rightarrow \mathbb{R}$ is then defined by

$$
\sigma(\Theta, \chi)=\sigma((\Upsilon, \Lambda),(\Phi, \Psi))=-\langle\Lambda, \Phi\rangle_{V}+\sigma_{1}(\Upsilon, \Psi)+\sigma_{2}(\Lambda, \Psi)
$$

where $\chi=(\Phi, \Psi)$ and $\Theta=(\Upsilon, \Lambda)$.

For the state $\mathcal{Z}(t)=\left(z(t), z_{t}(t)\right)$ in $\mathcal{H}$, we can subsequently write the system in the firstorder variational form

$$
\left\langle\mathcal{Z}_{t}(t), \chi\right\rangle_{\mathcal{V}^{*}, \mathcal{V}}+\sigma(\mathcal{Z}(t), \chi)=\langle\mathcal{B} u(t)+\mathcal{F}(t)+\mathcal{G}(\mathcal{Z}(t)), \chi\rangle_{\mathcal{V}^{*}, \mathcal{V}}
$$

where $\mathcal{F}(t)=(0, F(t)), \mathcal{G}(\mathcal{Z}(t))=\left(0, G\left(z(t), z_{t}(t)\right)\right)$ and $\mathcal{B} u(t)=(0, B u(t))$. As usual, the relation (15) must hold for all $\chi \in \mathcal{V}$. Finally, the weak form (15) is formally equivalent to the system

$$
\mathcal{Z}_{t}(t)=\mathcal{A Z}(t)+\mathcal{C}(t, \mathcal{Z}(t))
$$


in $\mathcal{V}^{*}$ where

$$
\mathcal{C}(t, \mathcal{Z}(t))=\mathcal{B} u(t)+\mathcal{F}(t)+\mathcal{G}(\mathcal{Z}(t))
$$

and

$$
\begin{gathered}
\operatorname{dom} \mathcal{A}=\left\{\Theta=(\Upsilon, \Lambda) \in \mathcal{H}: \Lambda \in V, A_{1} \Upsilon+A_{2} \Lambda \in H\right\} \\
\mathcal{A}=\left[\begin{array}{cc}
0 & I \\
-A_{1} & -A_{2}
\end{array}\right]
\end{gathered}
$$

\subsection{Model Well-Posedness}

In the previous discussion, the weak form of the coupled structural acoustic equations was written as an abstract first-order semilinear initial value problem with a state in $\mathcal{H}$. The nonlinear forcing term $\mathcal{C}(t, \mathcal{Z}(t))=\mathcal{B} u(t)+\mathcal{F}(t)+\mathcal{G}(\mathcal{Z}(t))$ however lies in $\mathcal{V}^{*}$ rather than $\mathcal{H}$ since the control term $B \in \mathcal{L}\left(U, V^{*}\right)$ defines the product space control term $\mathcal{B} u(t)=$ $(0, B u(t)) \in\{0\} \times V^{*} \subset V \times V^{*}=\mathcal{V}^{*}$. Hence the standard theory for abstract semilinear Cauchy problems does not apply directly, and the first step in the following discussion is the outline for arguments which can be used to extend the operator $\mathcal{A}$ to a space where the theory does apply. A more extensive discussion concerning the well-posedness of a linear problem of this type can be found in [15] and details for the following arguments can be found in that work.

The first step in determining the well-posedness of the system model is to argue that $\mathcal{A}$ generates a $C_{0}$-semigroup on $\mathcal{H}$. As noted earlier, the sesquilinear form $\sigma_{1}$ is $\mathrm{V}$-elliptic, continuous and symmetric while $\sigma_{2}$ is continuous and H-semielliptic. From the Lumer-Philips theorem (with further arguments found in [1] and pages 82-84 of [4]) this then implies that the operator $\mathcal{A}$ defined in (18) generates a $C_{0}$-semigroup on the state space $\mathcal{H}$. Moreover, the semigroup satisfies the exponential bound $|\mathcal{T}(t)| \leq e^{\omega t}$ for $t \geq 0$ (where in fact, $\omega=0$ due to the fact that $\mathcal{A}$ is dissipative as shown in [4]).

Since $\mathcal{B} u(t)$ lies in $\mathcal{V}^{*}$ rather than $\mathcal{H}$, the next step is to extend the semigroup $\mathcal{T}(t)$ on $\mathcal{H}$ to a semigroup $\tilde{\mathcal{T}}(t)$ on a larger space $\mathcal{W}^{*} \supset\{0\} \times V^{*}$ so as to be compatible with the forcing term (this is accomplished using "extrapolation space" ideas and arguments similar to those presented in $[6,7,22])$.

As detailed in [15], the space of interest is defined in terms of dom $\mathcal{A}^{*}$ where

$$
\begin{gathered}
\operatorname{dom} \mathcal{A}^{*}=\left\{\chi=(\Phi, \Psi) \in \mathcal{H} \mid \Psi \in V, A_{1}^{*} \Phi-A_{2}^{*} \Psi \in H\right\} \\
\mathcal{A}^{*} \chi=\left(\begin{array}{c}
-\Psi \\
A_{1}^{*} \Phi-A_{2}^{*} \Psi
\end{array}\right)
\end{gathered}
$$

Specifically, the space $\mathcal{W}=\left[\operatorname{dom} \mathcal{A}^{*}\right]$ is taken to be $\operatorname{dom} \mathcal{A}^{*}$ with the inner product

$$
\langle\Phi, \Psi\rangle_{\mathcal{W}}=\left\langle\left(\lambda_{0}-\mathcal{A}^{*}\right) \Phi,\left(\lambda_{0}-\mathcal{A}^{*}\right) \Psi\right\rangle_{\mathcal{H}}
$$

for some arbitrary but fixed $\lambda_{0}$ with $\lambda_{0}>\omega$ (recall that the original solution semigroup satisfies the bound $\left.|\mathcal{T}(t)| \leq e^{\omega t}\right)$. As proven in [7], the resulting $\mathcal{W}$ norm is equivalent to the graph norm corresponding to $\mathcal{A}^{*}$. Moreover, we have that $\{0\} \times V^{*} \subset \mathcal{W}^{*}=\left[\operatorname{dom} \mathcal{A}^{*}\right]^{*}$ (see [15] for details). 
From the definition of $\mathcal{A}^{*}$ and the equivalence of the $\mathcal{W}$ norm with the graph norm corresponding to $\mathcal{A}^{*}$, we can define $\tilde{\mathcal{A}} \Theta \in \mathcal{W}^{*}$ by

$$
(\tilde{\mathcal{A}} \Theta)(\chi)=\left\langle\Theta, \mathcal{A}^{*} \chi\right\rangle_{\mathcal{H}}
$$

for all $\Theta \in \mathcal{H}, \chi \in \mathcal{W}$. With this definition and the Riesz representation theorem, it is shown in [15] that $\tilde{A}$ is an extension of the original operator $\mathcal{A}$ from $\operatorname{dom} \mathcal{A} \subset \mathcal{H}$ to all of $\mathcal{H}$. Finally, as proven in [7], the operator $\tilde{\mathcal{A}}$ is the infinitesimal generator of a $C_{0}$-semigroup $\tilde{\mathcal{T}}(t)$ on $\mathcal{W}^{*}$ which is an extension of $\mathcal{T}(t)$ from $\mathcal{H}$ to $\mathcal{W}^{*}$.

In the corresponding linear problem, under reasonable regularity conditions on $t \mapsto u(t)$ and $t \mapsto F(t)$, one can immediately argue the existence of a unique strong solution to the system in terms of the extended semigroup $\tilde{\mathcal{T}}(t)$. For the semilinear problem of interest, however, the nonlinear nonhomogeneous terms must satisfy certain continuity criteria in order to obtain similar results. For example, if we let $X$ denote the reflexive Banach space $\mathcal{W}^{*}$ and assume that $\mathcal{C}:[0, T] \times X \rightarrow X$ defined in (17) is continuous in $t$ on $[0, T]$ and uniformly Lipschitz continuous on $X$, then the integral equation

$$
\mathcal{Z}(t)=\tilde{\mathcal{T}}(t) \mathcal{Z}_{0}+\int_{0}^{t} \tilde{\mathcal{T}}(t-s)\left(\begin{array}{c}
0 \\
B u(s)+F(s)+G(\mathcal{Z}(s))
\end{array}\right) d s
$$

is well-defined for $B u+F+G(\mathcal{Z}) \in L^{2}\left((0, T), V^{*}\right)$. Moreover, for $\mathcal{Z}(0)=\mathcal{Z}_{0}$, the solution $\mathcal{Z}(t)$ of (19) is a unique mild solution to (16) (see Theorem 1.2, page 184 of [27]). In addition, if $\mathcal{C}:[0, T] \times X \rightarrow X$ is Lipschitz continuous in both variables, then it follows from Theorem 1.6, page 189 of [27] that (19) provides the strong solution to (16) interpreted in the $\mathcal{W}^{*}$ sense.

The required continuity of the nonhomogeneous terms $B u$ and $F$ is demonstrated in [15] and hence the remaining question concerns the Lipschitz continuity of the nonlinear coupling term $G\left(z, z_{t}\right)=\left(0,-\rho_{f} \tilde{\phi}_{t}(w)\right)$. If we assume that the input terms $F$ and $B u$ are sufficiently smooth so as to assure the necessary continuity in $G\left(z, z_{t}\right)$, then our open loop nonlinear system is well-posed.

\subsection{Well-Posedness of the Closed Loop System}

The arguments leading to the well-posedness results for the linear and nonlinear open loop models can also be extended to the closed loop systems which result when the gains determined for a corresponding LQR problem are fed back into the system. In determining these gains, the perturbing force $\mathcal{F}$ is assumed to be periodic (this is a reasonable assumption since $\mathcal{F}$ models the exterior noise which in this problem is generated by the revolution of turboprop or turbofan blades).

Discussing first the linearized problem, the periodic LQR problem consists of finding $u \in$ $L^{2}(0, \tau ; U)$ which minimizes a quadratic cost functional of the form

$$
J(u)=\frac{1}{2} \int_{0}^{\tau}\left\{\langle\mathcal{Q Z}(t), \mathcal{Z}(t)\rangle_{\mathcal{H}}+\langle R u(t), u(t)\rangle_{U}\right\} d t
$$

subject to $\mathcal{Z}_{t}(t)=\mathcal{A Z}(t)+\mathcal{B} u(t)+\mathcal{F}(t)$ with $\mathcal{Z}(0)=\mathcal{Z}(\tau)$. Since $\mathcal{Z}=\left(\phi, w, \phi_{t}, w_{t}\right)$, the operator $\mathcal{Q}$ can be chosen so as to emphasize the minimization of particular state variables as well as to create windows that can be used to decrease state variations of certain frequencies. 
The control space $U$ is taken to be $\mathbb{R}^{s}$ if $s$ patches are used in the model, and it is assumed that the operator $R \in \mathcal{L}(U)$ is an $s \times s$ diagonal matrix where $r_{i i}>0, i=1, \cdots, s$ is the weight on the controlling voltage into the $i^{\text {th }}$ patch. In the case that $\mathcal{B}$ is bounded on $\mathcal{H}$, a complete feedback theory for this periodic problem can be given as discussed in [19]. This theory can be extended to also include the case of unbounded $\mathcal{B}$, i.e., $\mathcal{B} \in \mathcal{L}\left(U, \mathcal{V}^{*}\right)$, of interest here (see [5]). Under usual stabilizability and detectability assumptions on the system as well as standard assumptions on $\mathcal{Q}$, the optimal control is given by

$$
u(t)=-R^{-1} \mathcal{B}^{*}[\Pi \mathcal{Z}(t)-r(t)]
$$

where $\Pi \in \mathcal{L}\left(\mathcal{V}^{*}, \mathcal{V}\right)$ is the unique nonnegative self-adjoint solution of the algebraic Riccati equation

$$
\mathcal{A}^{*} \Pi+\Pi \mathcal{A}-\Pi \mathcal{B} R^{-1} \mathcal{B}^{*} \Pi+\mathcal{Q}=0 .
$$

Here $r$ is the unique $\tau$-periodic solution of

$$
\dot{r}(t)=-\left(\mathcal{A}^{*}-\Pi \mathcal{B} R^{-1} \mathcal{B}^{*}\right) r(t)+\Pi \mathcal{F}(t)
$$

and the optimal trajectory $\mathcal{Z}$ is the solution of

$$
\dot{\mathcal{Z}}(t)=\left(\mathcal{A}-\mathcal{B} R^{-1} \mathcal{B}^{*} \Pi\right) \mathcal{Z}(t)+\mathcal{B} R^{-1} \mathcal{B}^{*} r(t)+\mathcal{F}(t) .
$$

As discussed in [5] for the case when $\mathcal{B} \in \mathcal{L}\left(U, \mathcal{V}^{*}\right)$, one also finds that the operator $\mathcal{A}-\mathcal{B} R^{-1} \mathcal{B}^{*} \Pi$ generates an exponentially stable $C_{0}$-semigroup $\mathcal{S}(t)$ on the state space $\mathcal{H}$. From Corollary 10.6 , page 41 of [27], this implies that $\mathcal{A}^{*}-\Pi \mathcal{B} R^{-1} \mathcal{B}^{*}$ generates the corresponding adjoint semigroup $\mathcal{S}^{*}(t)$ on $\mathcal{H}^{*} \simeq \mathcal{H}$. The semigroup $\mathcal{S}(t)$ can then be extended through the extrapolation space techniques just discussed to a larger space $\tilde{\mathcal{W}}^{*} \supset\{0\} \times V^{*}$, and with reasonable regularity assumptions on $t \mapsto F(t)$, this implies the existence of solutions to the tracking equation (22) and closed loop system (23) for $r(0)=r_{0}$ and $\mathcal{Z}(0)=\mathcal{Z}_{0}$.

As discussed in greater detail in the next section where the corresponding finite dimensional control problem is considered, an effective strategy for controlling the original nonlinear system is to determine the gains for the linearized model and feed these back into the nonlinear system. This then yields the nonlinear closed loop system

$$
\dot{\mathcal{Z}}(t)=\left(\mathcal{A}-\mathcal{B} R^{-1} \mathcal{B}^{*} \Pi\right) \mathcal{Z}(t)+\mathcal{B} R^{-1} \mathcal{B}^{*} r(t)+\mathcal{F}(t)+\mathcal{G}((\mathcal{Z}(t))
$$

where again, $\mathcal{A}-\mathcal{B} R^{-1} \mathcal{B}^{*} \Pi$ generates the $C_{0}$-semigroup $\mathcal{S}(t)$ which can then be extended to $\tilde{\mathcal{W}}^{*}$. With the assumption that the input term $F$ is sufficiently smooth so as to assure the necessary continuity in nonhomogeneous terms, the closed loop nonlinear system is also well-posed. 


\section{System Approximation and the Finite Dimensional Control Problem}

The discussion thus far has centered around the infinite dimensional model for the structural acoustic system as well as issues concerning its well-posedness. However, in order to develop viable schemes for approximating the nonlinear system dynamics, estimating physical parameters, and determining control gains, appropriate finite dimensional approximations to the state variables $w$ and $\phi$ must be developed. For reasons discussed in [3], a Galerkin scheme was chosen and the potential and beam displacement were discretized in terms of spline and spectral expansions, respectively.

\subsection{System Approximation}

A tensored Legendre basis was used for the discretization of the acoustic velocity potential. Letting $P_{i}^{a}(x)$ and $P_{i}^{\ell}(y)$ denote the standard Legendre polynomials that have been scaled by transformation to the intervals $[0, a]$ and $[0, \ell]$, respectively, the basis functions $\left\{B_{i j}^{m}\right\}$ for the cavity were then defined as

$$
B_{i j}^{m}(x, y)=P_{i}^{a}(x) P_{j}^{\ell}(y) \quad \text { for } \quad i=0,1, \cdots, m_{x}, j=0,1, \cdots, m_{y}, i+j \neq 0,
$$

where $m=\left(m_{x}+1\right) \cdot\left(m_{y}+1\right)-1$. The condition $i+j \neq 0$ eliminates the constant function thus guaranteeing that the set of functions is suitable as a basis for the quotient space. The $m$ dimensional cavity approximating subspace is taken to be $H_{c}^{m}=\operatorname{span}\left\{B_{i}^{m}\right\}_{i=1}^{m}$ and the approximate cavity solution is given by

$$
\begin{aligned}
\phi^{N}(t, x, y) & =\sum_{i=1}^{m} \phi_{i}^{N}(t) B_{i}^{m}(x, y) \\
& =\sum_{j=0}^{m_{y}} \sum_{\substack{i=0 \\
i+j \neq 0}}^{m_{x}} \tilde{\phi}_{i j}^{N}(t) P_{i}^{a}(x) P_{j}^{\ell}(y) .
\end{aligned}
$$

Cubic splines were used as a basis for the beam displacement since they satisfy the smoothness requirement as well as being easily implemented when adapting to the fixed-end boundary conditions and patch discretizations. Letting $\left\{B_{i}^{n}\right\}_{i=1}^{n-1}$ denote the cubic splines which have been modified to satisfy the boundary conditions (see $[3,14]$ for details), the corresponding $n-1$ dimensional beam approximating subspace is given by $H_{b}^{n}=\operatorname{span}\left\{B_{i}^{n}\right\}_{i=1}^{n-1}$ and the approximate beam solution is taken to be

$$
w^{N}(t, x)=\sum_{i=1}^{n-1} w_{i}^{N}(t) B_{i}^{n}(x) .
$$

The approximating state space was then taken to be $H^{N}=H_{c}^{m} \times H_{b}^{n}$ where $N=m+n-1$, and the product space for the first order system is $\mathcal{H}^{N}=H^{N} \times H^{N}$. By restricting the infinite dimensional system (13) to $\mathcal{H}^{N} \times \mathcal{H}^{N}$, one obtains the nonlinear finite dimensional system

$$
\begin{aligned}
& M^{N} \dot{y}^{N}(t)=\tilde{\mathcal{A}}^{N}\left(y^{N}(t)\right)+\tilde{B}^{N} u(t)+\tilde{F}^{N}(t) \\
& M^{N} y^{N}(0)=\tilde{y}_{0}^{N}
\end{aligned}
$$


or equivalently

$$
\begin{aligned}
& \dot{y}^{N}(t)=\mathcal{A}^{N}\left(y^{N}(t)\right)+B^{N} u(t)+F^{N}(t) \\
& y^{N}(0)=\tilde{y}_{0}^{N}
\end{aligned}
$$

Explicit descriptions of the mass and stiffness operators $\bar{M}^{N}$ and $\tilde{\mathcal{A}}^{N}\left(y^{N}(t)\right)$ as well as detailed definitions of the control matrix $\tilde{B}^{N}$ and the force vector $\tilde{F}^{N}(t)$ can be found in [3, 13]. The vector $y^{N}(t)=\left(\phi_{1}^{N}(t), \cdots, \phi_{m}^{N}(t), w_{1}^{N}(t), \cdots, w_{n-1}^{N}(t), \dot{\phi}_{1}^{N}(t), \cdots, \dot{\phi}_{m}^{N}(t), \dot{w}_{1}^{N}(t), \cdots, \dot{w}_{n-1}^{N}(t)\right)^{T}$ contains the $2 N \times 1$ approximate state coefficients while $u(t)=\left(u_{1}(t), \cdots u_{s}(t)\right)^{T}$ contains the $s$ control variables. As detailed in [14], the nonlinearity in the operator $\tilde{\mathcal{A}}^{N}\left(y^{N}(t)\right)$ manifests itself in the dependence of the operator on the unknown coefficients $\left\{w_{j}(t)\right\}$.

\subsection{The Finite Dimensional Control Problem}

Due to the nonlinearity in the infinite dimensional system (13) and hence the finite dimensional matrix system (24), LQR feedback control results for problems with periodic forcing terms can not be directly applied as there were in [3]. Instead, the following strategy was adopted. The infinite dimensional system was linearized by replacing the nonlinear coupling term $\phi_{t}(t, x, w(t, x))$ by its linear component $\phi_{t}(t, x, 0)$ (this is equivalent to taking $G\left(z(t), z_{t}(t)\right)=0$ in (14) or $(\mathcal{G}(\mathcal{Z}(t))=0$ in (15) or (16)). This linearization is motivated by the assumption of small beam displacements which is inherent in the Euler-Bernoulli theory (for physically reasonable input forces, the beam displacements are of the order $10^{-5} \mathrm{~m}$ for the geometries of interest). The feedback gains for this approximate linearized system were calculated from a periodic LQR theory (see [3]) and were then fed back into the nonlinear problem to create a stable nonlinear closed loop control system.

To illustrate this control strategy, the LQR theory for problems with periodic input terms is briefly outlined. The resulting gains are then applied to the nonlinear problem of interest with the results being illustrated in an example.

\section{Linear Periodic Control Problem}

As discussed in [3], the approximation of the nonlinear coupling term $\phi_{t}(t, x, w(t, x))$ by its linear component, and the projection of the resulting system into the finite dimensional subspace $\mathcal{H}^{N} \times \mathcal{H}^{N}$ yields the linear finite dimensional Cauchy equation

$$
\begin{aligned}
& \dot{y}^{N}(t)=A^{N} y^{N}(t)+B^{N} u(t)+F^{N}(t) \\
& y^{N}(0)=y_{0}^{N}
\end{aligned}
$$

(this system can also be obtained by restricting the infinite dimensional system (14) with $G\left(z(t), z_{t}(t)\right)=0$ to $\left.\mathcal{H}^{N} \times \mathcal{H}^{N}\right)$. The components of the linear stiffness matrix can be found in $[3]$.

The periodic finite dimensional control problem is then to find $u \in L^{2}(0, \tau)$ which minimizes

$$
J^{N}(u)=\frac{1}{2} \int_{0}^{T}\left\{\left\langle Q^{N} y^{N}(t), y^{N}(t)\right\rangle_{\mathbf{R}^{N}}+\langle R u(t), u(t)\rangle_{\mathbf{R}^{0}}\right\} d t, \quad N=m+n-1
$$


where $y^{N}$ solves (25), $\tau$ is the period, $R$ is an $s \times s$ diagonal matrix and $r_{i i}>0, i=1, \cdots, s$ is the weight or penalty on the controlling voltage into the $i^{\text {th }}$ patch.

The nonnegative definite matrix $Q^{N}$ is chosen in a manner so as to to emphasize the minimization of particular state variables. From energy considerations as discussed in [3], an appropriate choice for $Q^{N}$ in this case is

$$
Q^{N}=M^{N} \mathcal{D}
$$

where $M^{N}$ is the mass matrix, and the diagonal matrix $\mathcal{D}$ is given by

$$
\mathcal{D}=\operatorname{diag}\left[d_{1} I^{m}, d_{2} I^{n-1}, d_{3} I^{m}, d_{4} I^{n-1}\right] \text {. }
$$

Here $I^{k}, k=m, n-1$, denotes a $k \times k$ identity and the parameters $d_{i}$ are chosen to enhance stability and performance of the feedback.

The optimal control is then given by

$$
u^{N}(t)=R^{-1}\left(B^{N}\right)^{T}\left[r^{N}(t)-\Pi^{N} y^{N}(t)\right]
$$

where $\Pi^{N}$ is the solution to the algebraic Riccati equation

$$
\left(A^{N}\right)^{T} \Pi^{N}+\Pi^{N} A^{N}-\Pi^{N} B^{N} R^{-1}\left(B^{N}\right)^{T} \Pi^{N}+Q^{N}=0 .
$$

For the regulator problem with periodic forcing function $F^{N}(t), r^{N}(t)$ must satisfy the linear differential equation

$$
\begin{aligned}
& \dot{r}^{N}(t)=-\left[A^{N}-B^{N} R^{-1}\left(B^{N}\right)^{T} \Pi^{N}\right]^{T} r^{N}(t)+\Pi^{N} F^{N}(t) \\
& r^{N}(0)=r^{N}(\tau)
\end{aligned}
$$

while the optimal trajectory is the solution to the linear differential equation

$$
\begin{aligned}
& \dot{y}^{N}(t)=\left[A^{N}-B^{N} R^{-1}\left(B^{N}\right)^{T} \Pi^{N}\right] y^{N}(t)+B^{N} R^{-1}\left(B^{N}\right)^{T} r^{N}(t)+F^{N}(t) \\
& y^{N}(0)=y^{N}(\tau) .
\end{aligned}
$$

The finite dimensional optimal control, Riccati solution, tracking equation and closed loop system can be compared with the original infinite dimensional relations given in (20), (21), (22) and (23), respectively. In order to guarantee the convergence $\Pi^{N} \rightarrow \Pi, r^{N} \rightarrow r$, and hence the convergence of $u^{N} \rightarrow u$, it is sufficient to impose various conditions on the original and approximate systems. These hypotheses include convergence requirements for the uncontrolled problem as well as the requirement that the approximation systems preserve stabilizability and detectability margins uniformly. A fully developed theory (see [5]) is available for the case when $\mathcal{F} \equiv 0$ (in this case the tracking variable $r$ does not appear in the solution) even when $\mathcal{B}$ is unbounded. However, the theory in [5] requires strong damping in the second-order system whereas the only damping in our system is the strong Kelvin-Voigt damping in the beam (damping in the cavity was omitted due to the relatively small dimensions involved). Although the convergence theory of [5] does not directly apply here, numerical tests indicate that convergence is obtained even though this system contains only weak or boundary damping. 


\section{Nonlinear Control Problem}

To extend these results to the nonlinear system of interest, the linear gains were calculated and fed back into the nonlinear system (24), thus yielding the suboptimal control

$$
u^{N}(t)=R^{-1}\left(B^{N}\right)^{T}\left[r^{N}(t)-\Pi^{N} y^{N}(t)\right]
$$

and the closed loop system

$$
\begin{aligned}
& \dot{y}^{N}(t)=\mathcal{A}^{N}\left(y^{N}(t)\right)-B^{N} R^{-1}\left(B^{N}\right)^{T} \Pi^{N} y^{N}(t)+B^{N} R^{-1}\left(B^{N}\right)^{T} r^{N}(t)+F^{N}(t) \\
& y^{N}(0)=y^{N}(\tau)
\end{aligned}
$$

The Riccati matrix $\Pi^{N}$ and tracking vector $r^{N}(t)$ are solutions to (26) and (27) which arise when formulating the corresponding LQR problem.

\section{Example: Nonlinear Control}

To illustrate the dynamics and effects of feedback control on a nonlinear system modeling a 2-D analogue of a 3-D experimental setup, a $6 \mathrm{~m}$ by $1 \mathrm{~m}$ cavity with a flexible beam at one end was considered (see Figure 6). The beam was assumed to have width and thickness $.1 \mathrm{~m}$ and $.005 \mathrm{~m}$, respectively, and the Young's modulus and beam density were taken to be $E=7.1 \times 10^{10} \mathrm{~N} / \mathrm{m}^{2}$ and $\rho_{b}=2700 \mathrm{~kg} / \mathrm{m}^{3}$. This yielded the stiffness parameter $E I=73.96 \mathrm{Nm}^{2}$ and linear mass density $\rho=1.35 \mathrm{~kg} / \mathrm{m}$. The damping parameter for the beam was chosen to be $c_{D} I=.001 \mathrm{~kg} \mathrm{~m}^{3} / \mathrm{sec}$. The speed of sound and atmospheric density inside the cavity were taken to be $c=343 \mathrm{~m} / \mathrm{sec}$ and $\rho_{f}=1.21 \mathrm{~kg} / \mathrm{m}^{3}$, respectively.

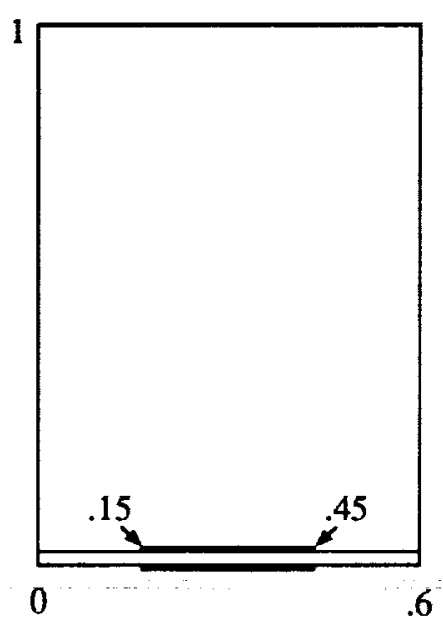

FIG. 6. Acoustic cavity with one centered $1 / 2$ length patch.

Several forcing functions modeling uniform (in space) periodic exterior sound sources were considered. In this example, the forcing function was taken to be

$$
f(t, x)=2.04 \sin (470 \pi t)
$$


which models a periodic plane wave with a root mean square (rms) sound pressure level of $117 \mathrm{~dB}$. The frequency of 235 hertz is approximately halfway between the first and fourth natural frequencies of the system (as shown in [11], these occur at 65.9 hertz and $387.8 \mathrm{hertz}$, respectively).

The dynamics of the uncontrolled system were approximated using 80 cavity basis functions $\left(m_{x}=m_{y}=8\right)$ and 11 beam basis functions $(n=12)$. The time interval of interest was taken to be $[0,16 / 235]$ which admitted 16 periods of the driving frequency, and time histories of the beam displacement at $X=.3$ and cavity pressure at $X=.3, Y=.1$ on this temporal interval are plotted in Figure 7.

The frequency plots of the uncontrolled beam displacement and cavity pressure in Figure 8 exhibit not only the driving frequency but also transient responses at $65.9,181.6,345.2,387.7$ and 519.5 hertz which are due to the natural frequencies of the coupled system (see [11] for a complete discussion of the dynamics and natural frequencies for the corresponding linearized system). In particular, the high energy response at 181.6 hertz indicates a strong excitation of the system at what corresponds to the frequency for the first mode of the uncoupled cavity (care must be taken when describing the dynamics of the system in terms of the undamped beam and cavity modes since the nonlinear coupling and beam damping yield system responses which differ somewhat from those of the isolated components). The presence of the multiple frequencies can also be seen in the time history plots of the uncontrolled beam displacement and cavity pressure in Figure 7.

Control was then implemented by using Potter's method to calculate the gains for the linearized system and feeding them back into the nonlinear system as discussed previously. The following results were obtained with an out-of-phase single pair (so as to create pure bending moments) of centered patches covering one half of the beam length as shown in Figure 6. The quadratic cost functional parameters were taken to be $d_{1}=d_{2}=d_{4}=1, d_{3}=10^{4}$ and $R=10^{-6}$ with $d_{3}$ chosen to have larger magnitude so as to more heavily penalize large. pressure variations.

Figure 9 contains a plot of the controlling voltage $u(t)$. As expected, it is periodic, and the magnitude remains below $25 \mathrm{~V}$ which is a physically reasonable voltage to apply to the piezoceramic patches.

The application of the controlling voltage resulted in a high frequency transient response and 168 cavity basis functions $\left(m_{x}=m_{y}=12\right)$ and 15 beam basis functions $(n=16)$ were needed to resolve the controlled system dynamics.

From Figure 7, it can be seen that the controlled responses undergo a transient phase of approximately three periods and then are maintained at a low magnitude throughout the rest of the time interval. By calculating the rms pressure levels, it was determined that at the point $(X, Y)=(.3, .1)$, the uncontrolled sound pressure level is $82.8 \mathrm{~dB}$ whereas the controlled sound pressure is reduced $15.7 \mathrm{~dB}$ to $67.1 \mathrm{~dB}$. The level of reduction becomes even more significant as one moves deeper into the cavity since the strong cavity excitation in the uncontrolled case yields high magnitude pressure oscillations near the back wall which are uniformly reduced by the application of the controlling voltage. Finally, it is noted that the relative reduction in pressure is more significant than the reduction in beam displacement. This is due to the heavier penalization of pressure fluctuations through the choices $d_{2}=1$ and $d_{3}=10^{4}$.

The frequency plots of the controlled responses (in Figure 8) show that the dominant response is now at the driving frequency of 235 hertz. They also demonstrate the presence of 
high frequency transient responses which are much more significant than those found in the uncontrolled case. This indicates that the interior pressure oscillations are reduced through two mechanisms when the controlling voltage is applied; the first is due to the reduced magnitude of the beam displacements while the second is due to the excitation of high frequency beam oscillations which couple less readily with the interior acoustic field. The combination of the two results in significantly reduced interior sound pressure levels.
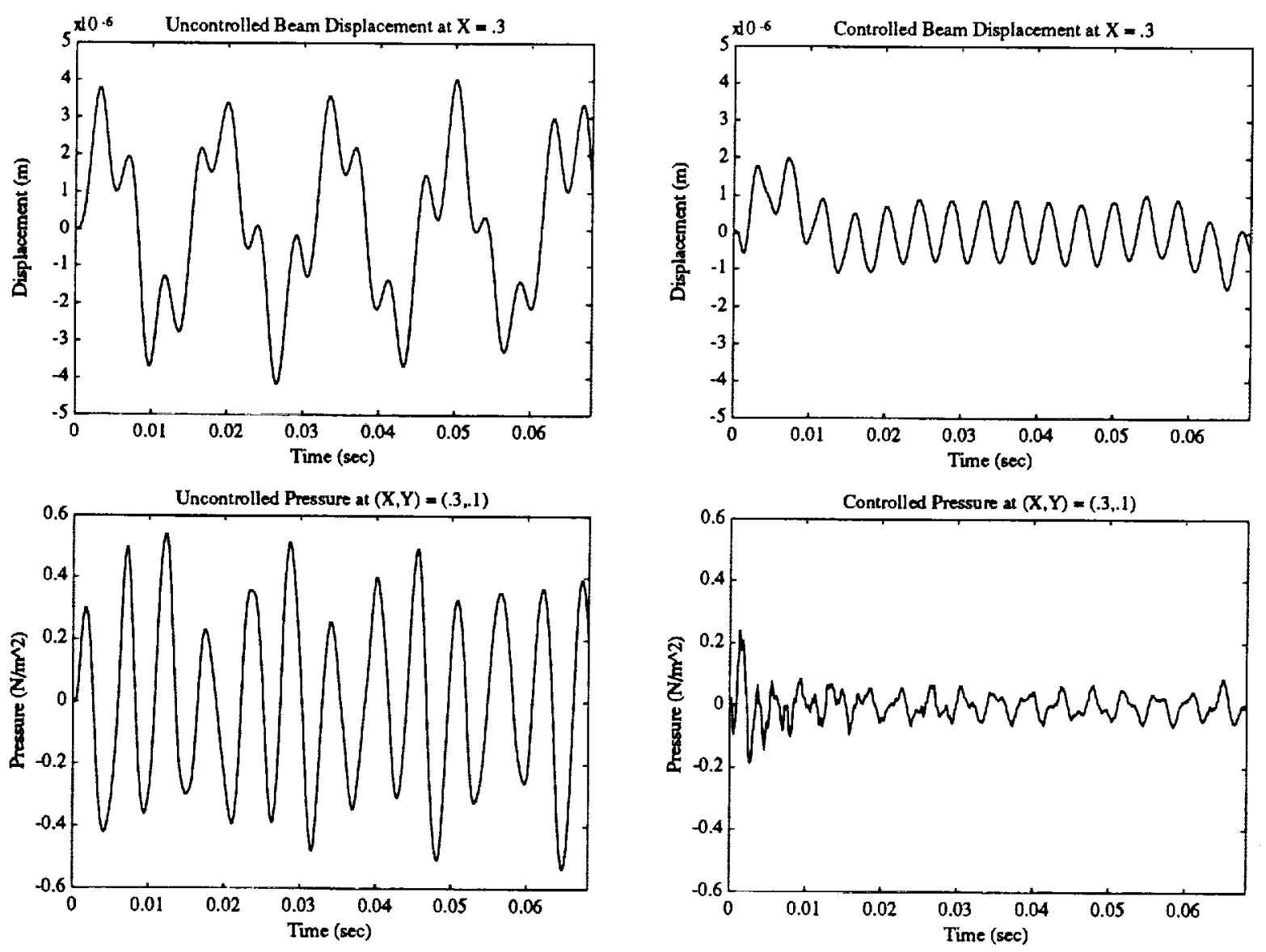

FIG. 7. Uncontrolled and controlled beam displacements and pressures at the points $X=.3$ and $(X, Y)=(.3, .1)$ throughout the time interval $[0,16 / 235]$. 



FIG. 8. Uncontrolled and controlled beam and cavity frequencies.

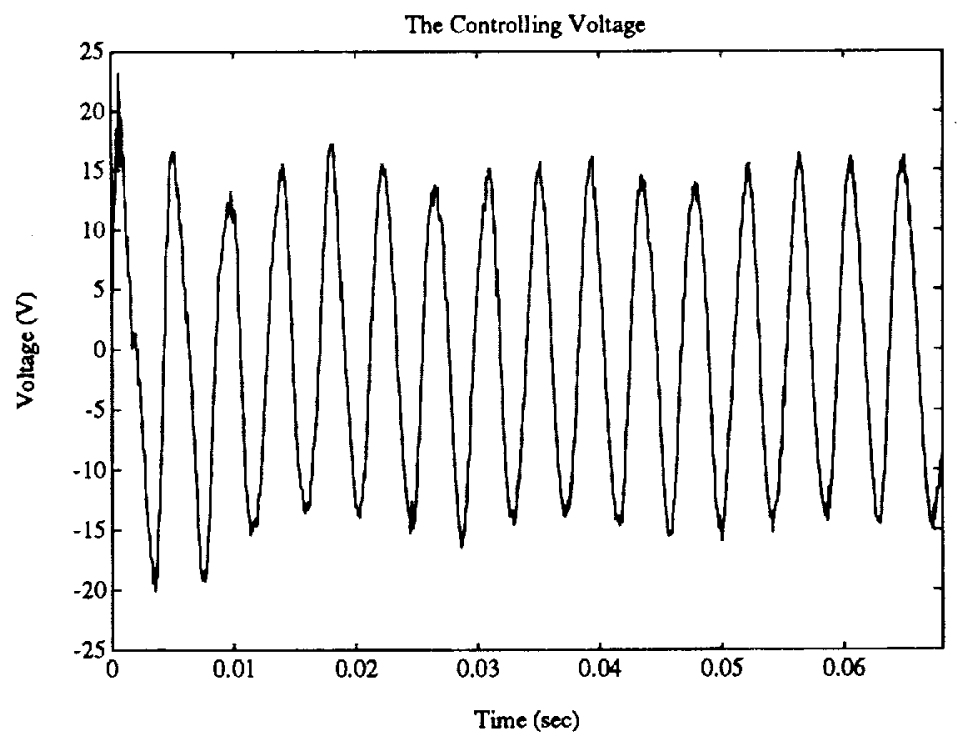

FIG. 9. The controlling voltage $u(t)$. 


\section{Conclusion}

In this paper, we have discussed several of the issues which are involved in using piezoceramic patches as actuators in a nonlinear structural acoustics application. The patches affect the dynamics of the coupled system by contributing external forces and moments to the structure when a voltage is applied, and the first part of the discussion is centered around a description of the interactions between the patches and an Euler-Bernoulli beam and a thin cylindrical shell. In this discussion, care was taken to distinguish between the passive (material) contributions, due to the added thickness and differing material properties of the patch and bonding layer, and the active (external) contributions which result from the strains which are produced when a voltage is applied to the patches.

As a result of the differing material properties and presence of the piezoceramic patches, the material and control parameters of the combined structure are piecewise constant in nature and hence lead to discontinuities in the moment and force resultants. This leads to difficulties in the strong form of the system equations when the moments are differentiated and is one motivation for using the weak or variational form where the derivatives are transferred onto the test functions. The weak form is also advantageous for many approximation schemes since it reduces the smoothness requirements for the basis elements. Finally, well-posedness issues were considered by posing the weak form in the context of sesquilinear forms.

Due to the nonlinearities arising in the coupling between the beam vibrations and the interior acoustic field, LQR feedback control results could not be directly applied to the problem. Instead, gains corresponding to the linearized problem were calculated and fed back into the nonlinear system. As demonstrated by the results in the example as well as the more extensive set of examples in [14], this strategy is very effective for this problem. This is partly due to the weakness of the nonlinearity. By comparing the nonlinear results reported here and in [14] with the corresponding linear ones in [11], one can see that qualitatively, the two sets agree closely. This can be explained by the fact that the beam displacements are very small and hence the linearized coupling terms quite accurately approximate the true nonlinear expressions.

\section{ACKNOWLEDGEMENTS:}

The authors would like to thank H.C. Lester and R.J. Silcox of the Acoustics Division, NASA Langley Research Center, for numerous discussions concerning the structural acoustic component of this work.

\section{References}

[1] H.T. Banks and J.A. Burns, Introduction to Control of Distributed Parameter Systems, Birkhäuser, to appear.

[2] H.T. Banks and F. Fakhroo, "Legendre-Tau Approximations for LQR Feedback Control of Acoustic Pressure Fields," Center for Research in Scientific Computation Technical Report, CRSC-TR92-5, North Carolina State University, Journal of Mathematical Systems, Estimation and Control, submitted. 
[3] H.T. Banks, W. Fang, R.J. Silcox and R.C. Smith, "Approximation Methods for Control of Acoustic/Structure Models with Piezoceramic Actuators," Journal of Intelligent Material Systems and Structures, 4(1), 1993, pp. 98-116.

[4] H.T. Banks and K. Ito, "A Unified Framework for Approximation in Inverse Problems for Distributed Parameter Systems," in Control-Theory and Advanced Technology 4(1), 1988, pp. 73-90.

[5] H.T. Banks and K. Ito, "Approximation in LQR Problems for Infinite Dimensional Systems with Unbounded Input Operators," Journal of Mathematical Systems, Estimation and Control, to be submitted.

[6] H.T. Banks, K. Ito and Y. Wang, "Well-Posedness for Damped Second Order Systems with Unbounded Input Operators," Journal of Mathematical Analysis and Applications, to be submitted.

[7] H.T. Banks, F. Kappel and C. Wang, "Weak Solutions and Differentiability for Size Structured Population Models," in Int. Series of Num. Math., Vol. 100, Birkhäuser, 1991, pp. 35-50.

[8] H.T. Banks, S.L. Keeling and R.J. Silcox, "Optimal Control Techniques for Active Noise Suppression," Proc. 27th IEEE Conf. on Decision and Control, Austin, Texas, 1988, pp. 2006-2011.

[9] H.T. Banks, S.L. Keeling, R.J. Silcox and C. Wang, "Linear Quadratic Tracking Problems in Hilbert Space: Applications to Optimal Active Noise Suppression," in Proc. $5^{\text {th }}$ IFAC Symp. on Control of DPS, (A. El-Jai and M. Amouroux, eds.), Perpignan, 1989, pp. 17-22.

[10] H.T. Banks, S.L. Keeling and C. Wang, "Linear Quadratic Tracking Problems in Infinite Dimensional Hilbert Spaces and a Finite Dimensional Approximation Framework," LCDC/CCS Rep. 88-28, October, 1988, Brown University.

[11] H.T. Banks, R.J. Silcox and R.C. Smith, "The Modeling and Control of Acoustic/Structure Interaction Problems via Piezoceramic Actuators: 2-D Numerical Examples," ASME Journal of Vibration and Acoustics, to appear.

[12] H.T. Banks and R.C. Smith, "Models for Control in Smart Material Structures," Center for Research in Scientific Computation Technical Report, CRSC-TR92-21, North Carolina State Univ., Proc. of the AMS/IMS/SIAM 10th Annual Joint Summer Conf. in the Math. Sciences, (Mt. Holyoke College, July, 1992), SIAM, to appear.

[13] H.T. Banks and R.C. Smith, "Feedback Control of Noise in a 2-D Nonlinear Structural Acoustics Model," Control-Theory and Advanced Technology, to be submitted.

[14] H.T. Banks and R.C. Smith, "Parameter Estimation in a 2-D Acoustic/Structure Interaction Model with Fully Nonlinear Coupling Conditions," Journal of Mathematical Systems, Estimation and Control, to be submitted. 
[15] H.T. Banks and R.C. Smith, "Well-Posedness of a Model for Structural Acoustic Coupling in a Cavity Enclosed by a Thin Cylindrical Shell," ICASE Report 93-10, Journal of Mathematical Analysis and Applications, submitted.

[16] H.T. Banks, R.C. Smith and Y. Wang, "Modeling Aspects for Piezoceramic Patch Activation of Shells, Plates and Beams," Center for Research in Scientific Computation Technical Report, CRSC-TR92-12, North Carolina State University, Quarterly of Applied Mathematics, to appear.

[17] H.T. Banks, Y. Wang, D.J. Inman and J.C. Slater, "Variable Coefficient Distributed Parameter System Models for Structures with Piezoceramic Actuators and Sensors," Proc. of the $31^{\text {st }}$ Conf. on Decision and Control, Tucson, AZ, Dec. 16-18, 1992, pp. 1803-1808.

[18] A.J. Bullmore, P.A. Nelson, A.R.D. Curtis and S.J. Elliott, "The Active Minimization of Harmonic Enclosed Sound Fields, Part II: A Computer Simulation," Journal of Sound and Vibration, 117(1), 1987, pp. 15-33.

[19] G. Da Prato, "Synthesis of Optimal Control for an Infinite Dimensional Periodic Problem," SIAM J. Control Opt., 25, 1987, pp. 706-714.

[20] J.J. Dosch, D.J. Inman and E. Garcia, "A Self-Sensing Piezoelectric Actuator for Collocated Control," J. Intell. Material Systems and Structures, 3, 1992, pp. 166-185.

[21] S.J. Elliott, A.R.D. Curtis, A.J. Bullmore and P.A. Nelson, "The Active Minimization of Harmonic Enclosed Sound Fields, Part III: Experimental Verification," Journal of Sound and Vibration, 117(1), 1987, pp. 35-58.

[22] A. Haraux, "Linear Semigroups in Banach Spaces," in Semigroups, Theory and Applications, $I I$ (H. Brezis, et al., eds.), Pitman Res. Notes in Math, Vol 152, Longman, London, 1986, pp. 93-135.

[23] A.W. Leissa, Vibration of Shells, NASA SP-288, 1973.

[24] H.C. Lester and C.R. Fuller, "Active Control of Propeller Induced Noise Fields Inside a Flexible Cylinder," AIAA Tenth Aeroacoustics Conference, Seattle, WA, 1986.

[25] S. Markuš, The Mechanics of Vibrations of Cylindrical Shells, Elsevier, NY, 1988.

[26] P.A. Nelson, A.R.D. Curtis, S.J. Elliott and A.J. Bullmore, "The Active Minimization of Harmonic Enclosed Sound Fields, Part I: Theory," Journal of Sound and Vibration, 117(1), 1987, pp. 1-13.

[27] A. Pazy, Semigroups of Linear Operators and Applications to Partial Differential Equations, Springer-Verlag, New York, 1983.

[28] R.J. Silcox, H.C. Lester and S.B. Abler, "An Evaluation of Active Noise Control in a Cylindrical Shell," NASA Technical Memorandum 89090, February 1987.

[29] J. Wloka, Partial Differential Equations, Cambridge Univ. Press, New York, 1987. 


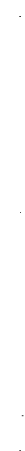




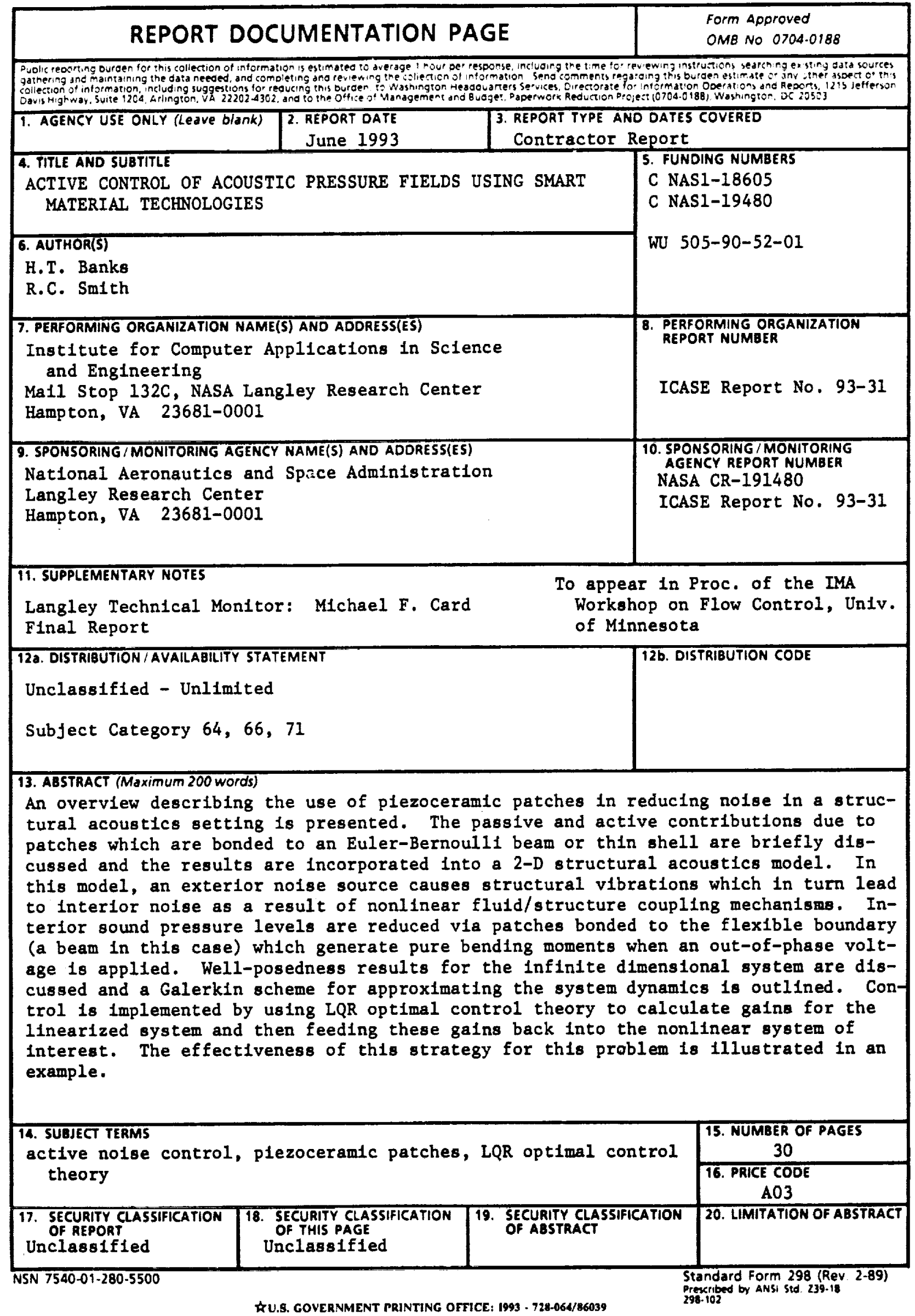

